\title{
Hesitant Fuzzy 2-Dimension Linguistic Programming Technique for Multidimensional Analysis of Preference for Multicriteria Group Decision Making
}

\author{
Xiaoyue Liu ${ }^{1, *}$ and Dawei Ju ${ }^{2}$ \\ 1 School of Information Technology \& Management, University of International Business and Economics, \\ Beijing 100029, China \\ 2 Beijing Engineering Research Center of Post Intelligent Equipment, Postal Scientific Research and \\ Planning Academy, Beijing 100096, China; i_dawei@126.com \\ * Correspondence: sxy880530@163.com; Tel.: +86-10-64495073
}

Citation: Liu, X.; Ju, D. Hesitant Fuzzy 2-Dimension Linguistic Programming Technique for Multidimensional Analysis of Preference for Multicriteria Group Decision Making. Mathematics 2021, 9 3196. https://doi.org/10.3390/ math9243196

Academic Editor: Tin-Chih Toly Chen

Received: 22 November 2021

Accepted: 7 December 2021

Published: 10 December 2021

Publisher's Note: MDPI stays neutral with regard to jurisdictional claims in published maps and institutional affiliations.

Copyright: (c) 2021 by the authors. Licensee MDPI, Basel, Switzerland. This article is an open access article distributed under the terms and conditions of the Creative Commons Attribution (CC BY) license (https:// creativecommons.org/licenses/by/ $4.0 /)$.

\begin{abstract}
The hesitant fuzzy 2-dimension linguistic element (HF2DLE) allows decision makers to express the importance or reliability of each term included in a hesitant fuzzy linguistic element as a linguistic term. This paper investigates a programming technique for multidimensional analysis of preference for hesitant fuzzy 2-dimension linguistic multicriteria group decision making. Considering the flexibility of HF2DLEs in expressing hesitant qualitative preference information, we first adopt HF2DLEs to depict both the evaluation values of alternatives and the truth degrees of alternative comparisons. To calculate the relative closeness degrees (RCDs) of alternatives, the Euclidean distances between HF2DLEs are defined. Based on RCDs and preference relations on alternatives, the group consistency and inconsistency indices are constructed, and a bi-objective hesitant fuzzy 2-dimension linguistic programming model is established to derive the criteria weights and positive and negative ideal solutions. Since the objective functions and partial constraint coefficients of the established model are HF2DLEs, an effective solution is developed, through which the RCDs can be calculated to obtain the individual rankings of alternatives. Furthermore, a single-objective assignment model is constructed to determine the best alternative. Finally, a case study is conducted to illustrate the feasibility of the proposed method, and its effectiveness is demonstrated by comparison analyses.
\end{abstract}

Keywords: multicriteria group decision making; hesitant fuzzy 2-dimension linguistic term set; linear programming technique for multidimensional analysis of preference; distance measure

\section{Introduction}

Multicriteria group decision making (MCGDM), a hot research topic in decision science, is used to select the best compromise solution from a feasible set of alternatives based on the preference information provided by a group of decision makers (DMs) with respect to multiple quantitative or qualitative criteria. Due to cost concerns, the limited knowledge of DMs, and the inherent subjective nature of human thinking, it is better for DMs to express their preferences in linguistic terms rather than numerical values, especially for qualitative criteria. To deal with linguistic information, Zadeh [1] introduced the fuzzy linguistic approach, which allows DMs to express their preferences with words or sentences in a natural or artificial language instead of numerical values. Many studies have focused on linguistic decision-making methods [2,3]. However, these studies are based on eliciting only one single linguistic term to express the DMs' preferences and thus cannot express the DMs' hesitations, because they may be thinking of several linguistic terms at the same time. For example, when evaluating a candidate, an interviewer may think that "they are higher than medium but not extremely good". In such cases, it is impossible to use a single linguistic term to express the linguistic evaluation. To solve this issue, combining a hesitant fuzzy set (HFS) [4] and linguistic terms, Rodríguez et al. [5] introduced the concept of a 
hesitant fuzzy linguistic term set (HFLTS), in which a set of consecutive linguistic terms is used to express the possible membership degrees of an element to a given set. For example, suppose that a linguistic term set (LTS) is $S=\left\{\dot{s}_{0}=\right.$ extremely poor, $\dot{s}_{1}=$ very poor, $\dot{s}_{2}=$ poor, $\dot{s}_{3}=$ medium, $\dot{s}_{4}=$ good, $\dot{s}_{5}=$ very good, $\dot{s}_{6}=$ extremely good $\}$; then, the above evaluation given by the interviewer could be denoted as a hesitant fuzzy linguistic element (HFLE) $h_{S}=\left\{\dot{s}_{4}, \dot{s}_{5}\right\}$.

Since HFLTS has remarkable power and efficiency in eliciting and representing linguistic preference information, it has attracted increasing attention from researchers in recent years [6], such as for operation and comparison methods of HFLEs [7-9], distance measures, similarity measures, and correlation coefficients between HFLEs [10-12], hesitant fuzzy linguistic aggregation techniques [13,14], hesitant fuzzy linguistic decision-making methods [15-17], and hesitant fuzzy linguistic preference relations [18-20]. Meanwhile, some extensions of HFLTS were introduced, such as extended HFLTS (EHFLTS) [21], double-hierarchy HFLTS (DHHFLTS) [22], the probabilistic linguistic term set (PLTS) [23], etc. Among these, considering the shortcoming that all linguistic terms included in an HFLE are equally important, Pang et al. [23] proposed the PLTS, in which DMs express the importance of each linguistic term with weight, using probability. However, sometimes the importance or reliability of linguistic terms cannot be expressed by numerical values. For example, in the process of a paper review, a reviewer provides their evaluation for a submitted paper as "I am certain this paper is good, but I have low certainty that it is very good". Based on the above-given LTS $S$, the HFLE is identified as $h_{S}=\left\{\dot{s}_{4}, \dot{s}_{5}\right\}$, where $\dot{s}_{4}$ and $\dot{s}_{5}$ are equally important. However, the reliability of $\dot{s}_{5}$ is low, so it should be less important or reliable than $\dot{s}_{4}$. To describe this type of information, the theory of the 2-dimension linguistic term set (2DLTS) [24] can be referenced, where one dimension uses a linguistic term to provide the evaluation of an alternative, and the other dimension uses a linguistic term to express the self-assessment of the reliability of the given evaluation result. Based on 2DLTS, Liu et al. [25] proposed the concept of a hesitant fuzzy 2-dimension linguistic term set (HF2DLTS), which allows DMs to use a linguistic term to express the importance or reliability of each linguistic term included in an HFLE. Let the above LTS $S$ be the first dimension LTS and $S^{(2)}=\left\{\ddot{s}_{0}=\right.$ extremely low certainty, $\ddot{s}_{1}=$ very low certainty, $\ddot{s}_{2}=$ low certainty, $\ddot{s}_{3}=$ certainty, $\ddot{s}_{4}=$ high certainty, $\ddot{s}_{5}=$ very high certainty, $\ddot{s}_{6}=$ extremely high certainty be the second dimension LTS. Then, for the above example, the hesitant fuzzy 2-dimension linguistic element (HF2DLE) is determined as $\hat{h}_{S}=\left\{\left(\dot{s}_{4}, \ddot{s}_{3}\right),\left(\dot{s}_{5}, \ddot{s}_{2}\right)\right\}$. To solve MCGDM problems in which the evaluation values of alternatives are denoted as HF2DLEs, Liu et al. [25] proposed several hesitant fuzzy 2-dimension linguistic aggregation operators.

There has been little research focused on developing and enriching the MCGDM methods under hesitant fuzzy 2-dimension linguistic environments. The linear programming technique for multidimensional analysis of preference (LINMAP) introduced by Srinivasan and Shocker [26] provides an efficient decision-making tool to address multicriteria decision making (MCDM) or MCGDM problems with preference relations on alternatives and unknown criteria weights. On the basis of the evaluation values of alternatives and a set of preference relations of pairwise comparisons between alternatives, LINMAP calculates the distances between alternatives and the positive ideal solution (PIS) to define the consistency and inconsistency indices. Based on this, a linear programming model is constructed to derive the PIS and criteria weights, and thus, the best compromise alternative as the solution that has the shortest distance to the PIS is determined. Compared with the well-known MCDM methods such as the technique for order preference by similarity to ideal solution (TOPSIS), elimination and choice translating reality (ELECTRE), and visekriterijumska optimizacija i kompromisno resenje (VIKOR; in Serbian) and the consistency analysis methods for preference relations, LINMAP considers the evaluation values of alternatives in addition to the preference relations of pairwise comparisons between alternatives, which allows them to be incomplete or even non-transitive. Moreover, it is obvious that LINMAP combines the objective and subjective information simultaneously, in which the criteria weights and PIS are determined objectively by constructing a programming model but not 
given by DMs in advance. Therefore, numerous LINMAP methods have been proposed to solve some real-life decision-making problems, such as energy project selection [27], undergraduate student recommendation [28], air-fighter selection [29], etc. However, in the traditional LINMAP method, all decision data are expressed by crisp numbers. Due to the increasing vagueness and uncertainty in decision making, the traditional LINMAP method cannot be directly employed to handle decision-making problems with fuzzy information, and some studies have extended the traditional LINMAP method to a variety of different fuzzy circumstances, such as fuzzy set [30], intuitionistic fuzzy set [29], interval-valued intuitionistic fuzzy set [28,30,31], hesitant fuzzy set [32], hesitant fuzzy linguistic term set [33], probabilistic linguistic term set [34], linguistic variable [35], interval type-2 fuzzy set [36], interval grey number [37], Pythagorean fuzzy set [38-40], interval-valued Pythagorean fuzzy set [39], and heterogeneous information [41-43]. To the best of our knowledge, there is no LINMAP method for hesitant fuzzy 2-dimension linguistic MCGDM. In this paper, considering the advantage of HF2DLTSs in representing DMs' hesitant qualitative preference information, both the evaluation values of alternatives and the truth degrees of alternative comparisons are expressed as HF2DLEs.

The TOPSIS method was proposed by Hwang and Yoon [44], which determines the most desirable alternative that has the shortest distance to PIS and the farthest distance to negative ideal solution (NIS). Due to its simplicity and comprehensibility, it has been widely used in diverse areas and extended to deal with fuzzy information [45-47]. The TOPSIS method considers two reference points, PIS and NIS, simultaneously, but it can only be used to solve MCDM problems with criteria weights known in advance. If the criteria weights are completely unknown or partially known, the TOPSIS method cannot be used. Therefore, in the application of TOPSIS, it is a critical issue to determine the weights of criteria accurately. Moreover, the PIS and NIS reference points involved in TOPSIS are directly determined by taking maximum and minimum evaluation values under each criterion, which may be unreasonable. From the above review of LINMAP, we know that the LINMAP method can determine criteria weights and PIS by constructing a programming model. However, the traditional LINMAP method only considers one reference point, i.e., PIS, and the consistency and inconsistency indices are defined based on the distances between alternatives and PIS. Motivated by the idea of TOPSIS, to take into account both PIS and NIS, this paper utilizes the relative closeness degree (RCD) instead of distance measure to construct the group consistency and inconsistency indices and hereby constructs a novel programming model to obtain criteria weights, as well as PIS and NIS, objectively. Therefore, the programming technique for multidimensional analysis of preference for hesitant fuzzy 2-dimension linguistic MCGDM proposed in this paper can well overcome the shortcomings of TOPSIS and LINMAP. First, we define the Euclidean distance measure between HF2DLEs, which is used to calculate the RCD of each alternative to hesitant fuzzy 2-dimension linguistic PIS (HF2DLPIS). Then, based on RCDs and preference relations on alternatives, the hesitant fuzzy 2-dimension linguistic group consistency and inconsistency indices are constructed, respectively, and a bi-objective hesitant fuzzy 2-dimension linguistic programming model is established to derive the criteria weights, HF2DLPIS, and hesitant fuzzy 2-dimension linguistic NIS (HF2DLNIS). Subsequently, for each DM, the RCDs of all feasible alternatives can be calculated, and the individual rankings of alternatives are derived according to the descending order of RCDs. Furthermore, a single-objective assignment model is constructed to generate the group ranking of alternatives, and the best alternative is determined. Finally, a case study about energy policy selection is conducted to illustrate the feasibility and effectiveness of the proposed method. The main contributions and features of this paper can be summarized as follows:

(1) Considering the fuzziness of the truth degrees of alternative comparisons, this is the first time that HF2DLEs have been adopted to describe both the evaluation values of alternatives and the truth degrees of alternative comparisons. Compared with HFLTS, the HF2DLTS allowing DMs to define the importance or reliability of each 
term as a linguistic term is more flexible in representing the hesitant qualitative preference information.

(2) To calculate the RCD of each alternative to HF2DLPIS, the Euclidean distance measure between HF2DLEs is first defined. Considering two reference points of HF2DLPIS and HF2DLNIS simultaneously, the RCD involved in TOPSIS is used to replace the distance measure in the traditional LINMAP method to construct the hesitant fuzzy 2-dimension linguistic group consistency and inconsistency indices.

(3) A bi-objective hesitant fuzzy 2-dimension linguistic programming model is constructed to determine the criteria weights, HF2DLPIS, and HF2DLNIS. Since both the objective functions and partial constraints' coefficients of the established model take the form of HF2DLEs, an effective solution method is technically developed to solve the constructed model.

(4) A case study about energy policy selection is conducted to illustrate the feasibility of the proposed method, and some comparative analyses with the aggregation operatorbased hesitant fuzzy 2-dimension linguistic MCGDM method, the hesitant fuzzy linguistic LINMAP method, and other existing MCGDM methods are conducted to show the effectiveness of the proposed method.

The remainder of this paper is organized as follows: In Section 2, we review some basic definitions related to HF2DLTS; Section 3 develops a hesitant fuzzy 2-dimension linguistic programming technique for multidimensional analysis of preference for MCGDM problems. In Section 4, a case study about energy policy selection is conducted to illustrate the feasibility of the proposed method, and its effectiveness is demonstrated by comparative analyses; Section 5 presents the conclusions, main features, and limitations of the proposed method, and discusses future research directions.

\section{Preliminaries}

To facilitate the following discussion, some definitions related to HF2DLTS are briefly reviewed in this section. The Euclidean distance measure between HF2DLEs is also introduced.

\subsection{Hesitant Fuzzy 2-Dimension Linguistic Term Set}

Let $S=\left\{s_{0}, s_{1}, \ldots, s_{g-1}\right\}$ be a finite and total ordered LTS with odd cardinality, where $g$ is the cardinality of LTS $S, s_{\alpha}(\alpha=0,1, \ldots, g-1)$ represents a possible value for a linguistic term and satisfies the following properties [48,49]:

(1) If $i>j$, then $s_{i}>s_{j}$ (i.e., $s_{i}$ is better than $s_{j}$ );

(2) There is a negation operator: $n e g\left(s_{i}\right)=s_{g-1-i}$;

(3) If $s_{i}>s_{j}$, then $\max \left(s_{i}, s_{j}\right)=s_{i}$;

(4) If $s_{i} \leq s_{j}$, then $\min \left(s_{i}, s_{j}\right)=s_{i}$.

In [50], the use of linguistic terms with odd cardinality was studied, in which the middle term of LTS represents "indifference", with the rest of the linguistic terms being placed symmetrically around it. This type of LTS has been widely used in decision making, evaluation processes, information retrieval, etc. According to a psychologist's suggestion, the LTS $S$ is better for providing $7 \pm 2$ linguistic terms, with less than 5 not being sufficiently informative and more than 9 being too much for a proper understanding of their differences [51]. To preserve all given information, $\mathrm{Xu}$ [52] extended the discrete LTS $S$ to a continuous one, $\bar{S}=\left\{s_{\alpha} \mid \alpha \in[0, g-1]\right\}$. In general, the linguistic term $s_{\alpha}\left(s_{\alpha} \in S\right)$ is given by DMs, while the extended linguistic term $s_{\alpha}\left(s_{\alpha} \in \bar{S}\right)$ only appears in computation. Moreover, there exist some relationships between linguistic terms and numerical values. Zhang and Qi [53] defined two equivalent transformation functions between linguistic terms and numerical values included in $[0,1]$. 
Definition 1 ([53]). Let $S=\left\{s_{0}, s_{1}, \ldots, s_{g-1}\right\}$ be an LTS and $r \in[0,1]$, then the numerical value $r$ that expresses the equivalent information to the extended linguistic term $s_{\alpha}\left(s_{\alpha} \in \bar{S}\right)$ is obtained with the following function $f$ :

$$
f:[0, g-1] \rightarrow[0,1], f\left(s_{\alpha}\right)=\frac{\alpha}{g-1}=r
$$

Additionally, the extended linguistic term $s_{\alpha}\left(s_{\alpha} \in \bar{S}\right)$ that expresses the equivalent information to the numerical value $r$ is obtained with the following function $f^{-1}$ :

$$
f^{-1}:[0,1] \rightarrow[0, g-1], f^{-1}(r)=s_{r \times(g-1)}=s_{\alpha}
$$

To depict the hesitant qualitative preference information, Rodríguez et al. [5] introduced the concept of HFLTS, and then, Liao and Xu [11] formalized the HFLTS mathematically as follows:

Definition 2 ([11]). Let $X$ be fixed and $S=\left\{s_{0}, s_{1}, \ldots, s_{g-1}\right\}$ be an LTS, then an HFLTS on $X$, $H_{S}$, can be denoted in the following mathematical form:

$$
H_{S}=\left\{<x, h_{S}(x)>\mid x \in X\right\},
$$

where $h_{S}(x)$, the possible membership degrees of the element $x \in X$ to $S$, is a set of consecutive linguistic terms in $S$ and can be denoted by $h_{S}(x)=\cup_{s(x) \in h_{S}(x)}\{s(x) \mid s(x) \in S\}$. For convenience, $h_{S}=h_{S}(x)$ is called the HFLE, and $H_{S}$ is the set of HFLEs.

In real-life decision making, DMs may be asked to give the evaluation values of alternatives, but also provide self-assessment on the reliability of the given evaluation results. To solve this issue, Zhu et al. [24] introduced the concept of 2DLTS.

Definition 3 ([24]). Let $S^{(1)}=\left\{\dot{s}_{0}, \dot{s}_{1}, \ldots, \dot{s}_{g-1}\right\}$ and $S^{(2)}=\left\{\ddot{s}_{0}, \ddot{s}_{1}, \ldots, \ddot{s}_{t-1}\right\}$ be two LTSs, where $g$ is the cardinality of $S^{(1)}, t$ is the cardinality of $S^{(2)}$ and $g$ and $t$ are independent. A 2DLTS is denoted as $\hat{s}=\left(\dot{s}_{a}, \ddot{s}_{b}\right)$, where $\dot{s}_{a} \in S^{(1)}$ represents the evaluation value of an alternative, and $\ddot{s}_{b} \in S^{(2)}$ represents the self-assessment on the reliability of $\dot{s}_{a}$.

As we clarified in the introduction, the HFLTS sometimes cannot describe the case in which the linguistic terms in an HFLE have different levels of importance or reliability. To more flexibly and comprehensively preserve the hesitant linguistic information, based on 2DLTS, Liu et al. [25] proposed the concept of HF2DLTS as follows:

Definition 4 ([25]). Let $X$ be fixed, $S^{(1)}=\left\{\dot{s}_{0}, \dot{s}_{1}, \ldots, \dot{s}_{g-1}\right\}$ and $S^{(2)}=\left\{\ddot{s}_{0}, \ddot{s}_{1}, \ldots, \ddot{s}_{t-1}\right\}$ be two LTSs, where $g$ is the cardinality of $S^{(1)}, t$ is the cardinality of $S^{(2)}$ and $g$ and $t$ are independent, then an HF2DLTS on $X, \hat{H}_{S}$, can be defined as

$$
\hat{H}_{S}=\left\{<x, \hat{h}_{S}(x)>\mid x \in X\right\},
$$

where $\hat{h}_{S}(x)=\cup_{\left(\dot{s}_{a}(x), \ddot{s}_{b}(x)\right) \in \hat{h}_{S}(x)}\left\{\left(\dot{s}_{a}(x), \ddot{s}_{b}(x)\right)\right\}, \dot{s}_{a}(x) \in S^{(1)}$ is the first-dimension linguistic information representing the possible membership degrees of the element $x \in X$ to the set $S^{(1)}$, and $\left\{\dot{s}_{a}(x) \in \hat{h}_{S}(x)\right\}$ is a set of consecutive linguistic terms in $S^{(1)} ; \ddot{s}_{b}(x) \in S^{(2)}$ is the seconddimension linguistic information describing the DM's subjective evaluation on the importance or reliability of $\dot{s}_{a}(x)$. For convenience, $\hat{h}_{S}=\hat{h}_{S}(x)$ is called the HF2DLE, and $\hat{H}_{S}$ is the set of HF2DLEs.

Comparing this with the dual hesitant fuzzy linguistic term set (DHFLTS) [54], DHFLTS uses a single linguistic term to describe the evaluation value of an alternative and two sets of crisp values in $[0,1]$ to denote the possible membership degrees and 
non-membership degrees of the alternative to the given linguistic term, while HF2DLTS uses HFLE, a set of consecutive linguistic terms, to describe the evaluation value to an alternative and provides the importance or reliability of each linguistic term included in an HFLE as a linguistic term.

\subsection{Some Basic Operations of HF2DLEs}

Let $\hat{h}_{S}=\cup_{\left(\dot{s}_{a}, \ddot{s}_{b}\right) \in \hat{h}_{S}}\left\{\left(\dot{s}_{a}, \ddot{s}_{b}\right)\right\}, \hat{h}_{S_{1}}=\cup_{\left(\dot{s}_{a_{1}}, \ddot{s}_{b_{1}}\right) \in \hat{h}_{S_{1}}}\left\{\left(\dot{s}_{a_{1}}, \ddot{s}_{b_{1}}\right)\right\}$ and $\hat{h}_{S_{2}}=\cup_{\left(\dot{s}_{a_{2}}, \ddot{s}_{b_{2}}\right) \in \hat{h}_{S_{2}}}$ $\left\{\left(\dot{s}_{a_{2}}, \ddot{s}_{b_{2}}\right)\right\}$ be any three HF2DLEs; then, the operational laws of HF2DLEs are defined as [25]:

$$
\begin{gathered}
\hat{h}_{S_{1}} \oplus \hat{h}_{S_{2}}=\cup_{\left.\left(\dot{s}_{a_{1}}, \ddot{s}_{b_{1}}\right) \in \hat{h}_{S_{1}}, \dot{s}_{a_{2}}, \ddot{s}_{b_{2}}\right) \in \hat{h}_{S_{2}}}\left\{\left(f^{-1}\left(f\left(\dot{s}_{a_{1}}\right)+f\left(\dot{s}_{a_{2}}\right)-f\left(\dot{s}_{a_{1}}\right) f\left(\dot{s}_{a_{2}}\right)\right), \min \left(\ddot{s}_{b_{1}}, \ddot{s}_{b_{2}}\right)\right)\right\}, \\
\hat{h}_{S_{1}} \otimes \hat{h}_{S_{2}}=\cup_{\left(\dot{s}_{a_{1}}, \ddot{s}_{b_{1}}\right) \in \hat{h}_{s_{1}},\left(\dot{s}_{a_{2}}, \ddot{s}_{b_{2}}\right) \in \hat{h}_{S_{2}}}\left\{\left(f^{-1}\left(f\left(\dot{s}_{a_{1}}\right) f\left(\dot{s}_{a_{2}}\right)\right), \min \left(\ddot{s}_{b_{1}}, \ddot{s}_{b_{2}}\right)\right)\right\}, \\
\lambda \hat{h}_{S}=\cup_{\left(\dot{s}_{a}, \ddot{s}_{b}\right) \in \hat{h}_{S}}\left\{\left(f^{-1}\left(1-\left(1-f\left(\dot{s}_{a}\right)\right)^{\lambda}\right), \ddot{s}_{b}\right)\right\}, \\
\left(\hat{h}_{S}\right)^{\lambda}=\cup_{\left(\dot{s}_{a}, \ddot{s}_{b}\right) \in \hat{h}_{S}}\left\{\left(f^{-1}\left(f\left(\dot{s}_{a}\right)^{\lambda}\right), \ddot{s}_{b}\right)\right\},
\end{gathered}
$$

where $f$ and $f^{-1}$ are the equivalent transformation functions between linguistic terms in $S^{(1)}$ and numerical values in $[0,1]$ defined in Definition 1 , and $\lambda$ is a non-negative real number.

To compare any two HF2DLEs, Liu et al. [25] proposed the score function of HF2DLE.

Definition 5 ([25]). Let $\hat{h}_{S}=\cup_{\left(\dot{s}_{a}, \ddot{s}_{b}\right) \in \hat{h}_{S}}\left\{\left(\dot{s}_{a}, \ddot{s}_{b}\right)\right\}$ be an HF2DLE; then, the score function of $\hat{h}_{S}$ is defined as follows:

$$
S\left(\hat{h}_{S}\right)=\frac{1}{\# \hat{h}_{S}} \sum_{\left(\dot{s}_{a}, \ddot{s}_{b}\right) \in \hat{h}_{S}}\left(f\left(\dot{s}_{a}\right) f\left(\ddot{s}_{b}\right)\right),
$$

where $\# \hat{h}_{S}$ is the number of elements in HF2DLE $\hat{h}_{S}$; fis the function of transforming linguistic terms into numerical values.

If the score values of any two HF2DLEs are equal, they cannot be compared. To solve this issue, on the basis of score function, we define the variance function of HF2DLE and repropose the comparison laws of HF2DLEs.

Definition 6. Let $\hat{h}_{S}=\cup_{\left(\dot{s}_{a}, \ddot{s}_{b}\right) \in \hat{h}_{S}}\left\{\left(\dot{s}_{a}, \ddot{s}_{b}\right)\right\}$ be an HF2DLE, then the variance function of $\hat{h}_{S}$ is defined as follows:

$$
V\left(\hat{h}_{S}\right)=\frac{1}{\# \hat{h}_{S}} \sum_{\left(\dot{s}_{a}, \ddot{s}_{b}\right) \in \hat{h}_{S}}\left(f\left(\dot{s}_{a}\right) f\left(\ddot{s}_{b}\right)-S\left(\hat{h}_{S}\right)\right)^{2},
$$

where is the score value of $\hat{h}_{S}$. For any two HF2DLEs, $\hat{h}_{S_{1}}$ and $\hat{h}_{S_{2}}$, the comparison laws of HF2DLEs are given as

(1) if $S\left(\hat{h}_{S_{1}}\right)>S\left(\hat{h}_{S_{2}}\right)$, then $\hat{h}_{S_{1}}>\hat{h}_{S_{2}}$;

(2) if $S\left(\hat{h}_{S_{1}}\right)=S\left(\hat{h}_{S_{2}}\right)$, then

(a) if $V\left(\hat{h}_{S_{1}}\right)>V\left(\hat{h}_{S_{2}}\right)$, then $\hat{h}_{S_{1}}<\hat{h}_{S_{2}}$;

(b) if $V\left(\hat{h}_{S_{1}}\right)=V\left(\hat{h}_{S_{2}}\right)$, then $\hat{h}_{S_{1}}=\hat{h}_{S_{2}}$.

The distance measure is essential for HF2DLEs, and it is also a key point in LINMAP to calculate the distance between each alternative and PIS (NIS) to construct the inconsistency and consistency indices. Hence, we propose the Euclidean distance measure between HF2DLEs. 
Definition 7. Let $\hat{h}_{S_{1}}=\cup_{\left(\dot{s}_{a_{1}}, \ddot{s}_{b_{1}}\right) \in \hat{h}_{S_{1}}}\left\{\left(\dot{s}_{a_{1}}, \ddot{s}_{b_{1}}\right)\right\}$ and $\hat{h}_{S_{2}}=\cup_{\left(\dot{s}_{a_{2}}, \ddot{s}_{b_{2}}\right) \in \hat{h}_{S_{2}}}\left\{\left(\dot{s}_{a_{2}}, \ddot{s}_{b_{2}}\right)\right\}$ be any two HF2DLEs; then, the Euclidean distance measure between them can be defined as

$$
d\left(\hat{h}_{S_{1}}, \hat{h}_{S_{2}}\right)=\sqrt{\frac{1}{L} \sum_{l=1}^{L}\left(\eta_{1}^{(l)}-\eta_{2}^{(l)}\right)^{2}},
$$

where $\eta_{1}^{(l)}$ and $\eta_{2}^{(l)}$ are the $l$-th smallest element of $\left\{f\left(\dot{s}_{a_{1}}\right) \cdot f\left(\ddot{s}_{b_{1}}\right) \mid\left(\dot{s}_{a_{1}}, \ddot{s}_{b_{1}}\right) \in \hat{h}_{S_{1}}\right\}$ and $\left\{f\left(\dot{s}_{a_{2}}\right)\right.$. $\left.f\left(\ddot{s}_{b_{2}}\right) \mid\left(\dot{s}_{a_{2}}, \ddot{s}_{b_{2}}\right) \in \hat{h}_{S_{2}}\right\}$, fis the function transforming linguistic terms into numerical values; $L=\max \left\{\# \hat{h}_{S_{1}}, \# \hat{h}_{S_{2}}\right\}, \# \hat{h}_{S_{1}}$ and $\# \hat{h}_{S_{2}}$ are the numbers of elements in $\hat{h}_{S_{1}}$ and $\hat{h}_{S_{2}}$. If $\# \hat{h}_{S_{1}} \neq \# \hat{h}_{S_{2_{2}}}$ we should extend the shorter one by adding the same value several times in it until both of them have the same length, such as if $\# \hat{h}_{S_{1}}>\# \hat{h}_{S_{2}}$, then we can add $\# \hat{h}_{S_{1}}-\# \hat{h}_{S_{2}}$ linguistic terms to $\hat{h}_{S_{2}}$. The added linguistic term is the smallest one in the first dimension of $\hat{h}_{S_{2}}\left(\dot{s}_{a_{2}} \in \hat{h}_{S_{2}}\right)$, and the importance of each added linguistic term is $\ddot{s}_{0}$.

It can be examined that for any three HF2DLEs, $\hat{h}_{S_{1}}=\cup_{\left(\dot{s}_{a_{1}}, \ddot{s}_{b_{1}}\right) \in \hat{h}_{S_{1}}}\left\{\left(\dot{s}_{a_{2}}, \ddot{s}_{b_{1}}\right)\right\}, \hat{h}_{S_{2}}=$ $\cup_{\left(\dot{s}_{a_{2}}, \ddot{s}_{b_{2}}\right) \in \hat{h}_{s_{2}}}\left\{\left(\dot{s}_{a_{2}}, \ddot{s}_{b_{2}}\right)\right\}$, and $\hat{h}_{S_{3}}=\cup_{\left(\dot{s}_{a_{3}}, \ddot{s}_{b_{3}}\right) \in \hat{h}_{S_{3}}}\left\{\left(\dot{s}_{a_{3}}, \ddot{s}_{b_{3}}\right)\right\}$, the Euclidean distance measure between HF2DLEs satisfies the following fundamental properties:

(1) Distance in the range of [0, 1]: $0 \leq d\left(\hat{h}_{S_{1}}, \hat{h}_{S_{2}}\right) \leq 1$;

(2) Symmetry: $d\left(\hat{h}_{S_{1}}, \hat{h}_{S_{2}}\right)=d\left(\hat{h}_{S_{2}}, \hat{h}_{S_{1}}\right)$;

(3) Transitivity: $d\left(\hat{h}_{S_{1}}, \hat{h}_{S_{2}}\right) \leq d\left(\hat{h}_{S_{1}}, \hat{h}_{S_{3}}\right)$ and $d\left(\hat{h}_{S_{2}}, \hat{h}_{S_{3}}\right) \leq d\left(\hat{h}_{S_{1}}, \hat{h}_{S_{3}}\right)$, if $\hat{h}_{S_{1}} \leq \hat{h}_{S_{2}} \leq \hat{h}_{S_{3}}$.

\section{Hesitant Fuzzy 2-Dimension Linguistic Programming Technique for Multidimensional Analysis of Preference for MCGDM}

As presented in the introduction, HF2DLTS is powerful in representing the DMs' hesitant qualitative preference information, and thus can be used in many different scenarios. In this section, motivated by the idea of LINMAP, a novel programming method is proposed for hesitant fuzzy 2-dimension linguistic MCGDM.

Suppose that there are $q$ DMs $D_{p}(p=1,2, \ldots, q)$ who are invited to evaluate the alternative $A_{i}(i=1,2, \ldots, m)$ on criterion $C_{j}(j=1,2, \ldots, n)$. Under a vague and uncertain environment, the expert interview method is used to collect the DMs' preference information, in which DMs may hesitate among several linguistic terms, and the reliability of each term is different, for example, if the DM $D_{1}$ feels that they are certain the alternative $A_{1}$ is good but have low certainty that it is very good with respect to criterion $C_{1}$. To preserve such linguistic preference information, HF2DLEs are adopted to express the rating of alternative $A_{i}(i=1,2, \ldots, m)$ on criterion $C_{j}(j=1,2, \ldots, n)$ provided by DM $D_{p}(p=1,2, \ldots, q)$ and denoted as $\hat{h}_{S_{i j}}^{(p)}=\cup_{\left(\dot{s}_{a_{i j}}^{(p)}, \ddot{s}_{b_{i j}}^{(p)}\right) \in \hat{h}_{S_{i j}}^{(p)}}\left\{\left(\dot{s}_{a_{i j}}^{(p)}, \ddot{s}_{b_{i j}}^{(p)}\right)\right\}$, where $\dot{s}_{a_{i j}}^{(p)} \in S^{(1)}$ is the first-dimension linguistic information representing the possible membership degrees of alternative $A_{i}(i=1,2, \ldots, m)$ to criterion $C_{j}(j=1,2, \ldots, n)$ provided by DM $D_{p}(p=1,2, \ldots, q)$ based on $S^{(1)}=\left\{\dot{s}_{0}, \dot{s}_{1}, \ldots, \dot{s}_{g-1}\right\}$, and $\ddot{s}_{b_{i j}}^{(p)} \in S^{(2)}$ is the seconddimension linguistic information, representing the importance or reliability of $\dot{s}_{a_{i j}}^{(p)}$ provided by DM $D_{p}(p=1,2, \ldots, q)$ based on $S^{(2)}=\left\{\ddot{s}_{0}, \ddot{s}_{1}, \ldots, \ddot{s}_{t-1}\right\}$. Hence, the hesitant fuzzy 2-dimension linguistic MCGDM problem can be concisely expressed in a matrix format as $\hat{H}_{S}^{p}=\left(\hat{h}_{S_{i j}}^{p}\right)_{m \times n}(p=1,2, \ldots, q)$.

The preference relations of pairwise comparisons between alternatives provided by $\mathrm{DM} D_{p}(p=1,2, \ldots, q)$ are denoted as $\Omega^{p}=\left\{<(k, i), \hat{h}_{S}^{(p)}(k, i)>\mid A_{k} \succeq_{p} A_{i} ; k, i=\right.$ $1,2, \ldots, m\}$ with a hesitant fuzzy 2-dimension linguistic truth degree $\hat{h}_{S}^{(p)}(k, i)$, where $(k, i)$ expresses an ordered pair of alternatives $A_{k}$ and $A_{i}$ that DM $D_{p}$ prefers $A_{k}$ to $A_{i}$ (denoted 
by $\left.A_{k} \succeq_{p} A_{i}\right)(p=1,2, \ldots, q ; k, i=1,2, \ldots, m)$, and $\hat{h}_{S}^{(p)}(k, i)$ is an HF2DLE. The preference relations $\Omega^{p}$ are given by pairwise comparisons between alternatives as a whole rather than each criterion. Usually, DMs would not be able to specify pairwise comparisons of all alternatives and may only give pairwise comparisons of partial alternatives. Thus, the preference relations of pairwise comparisons between alternatives are usually incomplete.

Denote the weight vector of criteria by $w=\left(w_{1}, w_{2}, \ldots, w_{n}\right)^{T}$, where $w_{j}$ is the weight of criterion $C_{j}(j=1,2, \ldots, n)$ satisfying $\sum_{j=1}^{n} w_{j}=1$ and $w_{j} \geq 0$. In this paper, $w$ is incompletely known and needs to be determined. Let $\Lambda=\left\{w \mid \sum_{j=1}^{n} w_{j}=1, w_{j} \geq \varepsilon, j=\right.$ $1,2, \ldots, n\}$, where $\varepsilon>0$ is a sufficiently small positive number which ensures that the weights generated are not zero. The incomplete weight information structure can be expressed in the five basic relations among criteria weights, which are denoted by subsets $\Lambda_{s}(s=1,2,3,4,5)$ of the weight vector in $\Lambda$ (see [55-57] for details). In reality, usually, the preference information structure $\Lambda$ of criterion weights may consist of several subsets of the above basic subsets $\Lambda_{s}(s=1,2,3,4,5)$. Thus, the hesitant fuzzy 2-dimension linguistic MCGDM considered in this paper is used to rank all feasible alternatives according to the hesitant fuzzy 2-dimension linguistic decision matrices $\hat{H}_{S}^{p}=\left(\hat{h}_{S_{i j}}^{p}\right)_{m \times n}(p=1,2, \ldots, q)$, the incomplete preference relations on alternatives $\Omega^{p}(p=1,2, \ldots, q)$, and the incomplete weight information on criteria $\Lambda$.

\subsection{Hesitant Fuzzy 2-Dimension Linguistic Group Inconsistency and Consistency Indices}

Suppose that HF2DLPIS is $\hat{h}_{S}^{+}=\left(\hat{h}_{S_{1}}^{+}, \hat{h}_{S_{2}}^{+}, \ldots, \hat{h}_{S_{n}}^{+}\right)$, and HF2DLNIS is $\hat{h}_{S}^{-}=\left(\hat{h}_{S_{1}}^{-}, \hat{h}_{S_{2}}^{-}, \ldots\right.$, $\left.\hat{h}_{S_{n}}^{-}\right)$, which are unknown a priori and need to be determined. $\left.\hat{h}_{S_{j}}^{+}=\cup_{\left(\dot{s}_{a_{j}}^{+}, \ddot{s}_{j}\right.}^{+}\right) \in \hat{h}_{s_{j}}^{+}\left\{\left(\dot{s}_{a_{j}}^{+}, \ddot{s}_{b_{j}}^{+}\right)\right\}$ and $\hat{h}_{S_{j}}^{-}=\cup_{\left(\dot{s}_{a_{j}}^{-}, \ddot{s}_{b_{j}}^{-}\right)} \in \hat{h}_{s_{j}}^{-}\left\{\left(\dot{s}_{a_{j}}^{-}, \ddot{s}_{b_{j}}^{-}\right)\right\}$are the best and worst ratings on criterion $C_{j}(j=1,2, \ldots, n)$. For DM $D_{p}(p=1,2, \ldots, q)$, who uses HF2DLEs to evaluate alternative $A_{i}(i=1,2, \ldots, m)$ of all the criteria, the hesitant fuzzy 2-dimension linguistic evaluation vector for each alternative can be obtained. We evaluate this kind of hesitant fuzzy 2-dimension linguistic evaluation vector $\hat{h}_{S_{i}}^{p}$ for DM $D_{p}(p=1,2, \ldots, q)$ with respect to alternative $A_{i}(i=1,2, \ldots, m)$ as $\hat{h}_{S_{i}}^{p}=\left(\hat{h}_{S_{i 1}}^{p}, \hat{h}_{S_{i 2}}^{p}, \ldots, \hat{h}_{S_{i n}}^{p}\right)$. According to Equation (11), the weighted average of the squared Euclidean distance between alternative $A_{i}(i=1,2, \ldots, m)$ and HF2DLPIS $\hat{h}_{S}^{+}$for $\mathrm{DM} D_{p}(p=1,2, \ldots, q)$ can be defined as follows:

$$
d_{i}^{p+}=d\left(\hat{h}_{S_{i}}^{p}, \hat{h}_{S}^{+}\right)=\sum_{j=1}^{n} w_{j} \sum_{l=1}^{L} \frac{\left(\eta_{i j}^{(l)}-\eta_{j+}^{(l)}\right)^{2}}{L}, p=1,2, \ldots, q ; i=1,2, \ldots, m,
$$

where $w=\left(w_{1}, w_{2}, \ldots, w_{n}\right)^{T}$ is the weight vector of criteria which needs to be determined; $\eta_{i j}^{(l)}$ and $\eta_{j+}^{(l)}$ are the $l$-th smallest element of $\left\{f\left(\dot{s}_{a_{j}}^{p}\right) \cdot f\left(\ddot{s}_{b_{j}}^{p}\right) \mid\left(\dot{s}_{a_{j}}^{p} \ddot{s}_{b_{j}}^{p}\right) \in \hat{h}_{S_{j}}^{p}\right\}$ and $\left\{f\left(\dot{s}_{a_{j}}^{+}\right) \cdot f\left(\ddot{s}_{b_{j}}^{+}\right) \mid\left(\dot{s}_{a_{j}}^{+} \ddot{s}_{b_{j}}^{+}\right) \in \hat{h}_{S_{j}}^{+}\right\}, f$ is the function transforming linguistic terms into numerical values, and $L$ is the number of elements in $\hat{h}_{S_{i j}}^{p}$. Here, for simplicity, we make the number of elements in all HF2DLEs equal.

Similarly, the weighted average of squared Euclidean distance between alternative $A_{\mathrm{i}}(i=1,2, \ldots, m)$ and HF2DLNIS $\hat{h}_{S}^{-}$for $\mathrm{DM} D_{p}(p=1,2, \ldots, q)$ is obtained as

$$
d_{i}^{p-}=d\left(\hat{h}_{S_{i}}^{p}, \hat{h}_{S}^{-}\right)=\sum_{j=1}^{n} w_{j} \sum_{l=1}^{L} \frac{\left(\eta_{i j}^{(l)}-\eta_{j-}^{(l)}\right)^{2}}{L}, p=1,2, \ldots, q ; i=1,2, \ldots, m,
$$

where $\eta_{j-}^{(l)}$ is the $l$-th smallest element of $\left\{f\left(\dot{s}_{a_{j}}^{-}\right) \cdot f\left(\ddot{s}_{b_{j}}^{-}\right) \mid\left(\dot{s}_{a_{j}}^{-} \ddot{s}_{b_{j}}^{-}\right) \in \hat{h}_{S_{j}}^{-}\right\}$. 
Based on the above distances, the RCD of alternative $A_{i}(i=1,2, \ldots, m)$ to HF2DLPIS $\hat{h}_{S}^{+}$for $\mathrm{DM} D_{p}(p=1,2, \ldots, q)$ can be determined as follows:

$$
R_{i}^{p}=\frac{d_{i}^{p-}}{d_{i}^{p-}+d_{i}^{p+}}, p=1,2, \ldots, q ; i=1,2, \ldots, m
$$

If the criterion weights, HF2DLPIS and HF2DLNIS are already given by DMs, then $R_{k}^{p}$ and $R_{i}^{p}$ can be calculated according to Equation (14), and the individual ranking of all feasible alternatives can be determined according to the descending order of RCDs. However, in this paper, it is conceived that the criteria weights HF2DLPIS and HF2DLNIS are unknown a priori. Instead, to determine these unknown factors objectively, the group consistency and inconsistency indices are defined, based on which a programming model is constructed.

For each $(k, i) \in \Omega^{p}$, if $R_{k}^{p} \geq R_{i}^{p}$, then $A_{k}$ is better than $A_{i}$, the objective ranking of alternatives $A_{k}$ and $A_{i}$ determined by $R_{k}^{p}$, and $R_{i}^{p}$ is consistent with the subjective preference given by the DM $D_{p}$. Conversely, if $R_{k}^{p}<R_{i}^{p}$, then the objective ranking of alternatives $A_{k}$ and $A_{i}$ determined by $R_{k}^{p}$, and $R_{i}^{p}$ is inconsistent with the subjective preference given by DM $D_{p}$. Therefore, there exist some deviations between the objective rankings and subjective preferences provided by DMs. To measure such deviation, the following hesitant fuzzy 2-dimension linguistic group inconsistency and consistency indices are defined based on RCDs and preference relations on alternatives.

The inconsistency index $\theta_{k i}^{p}$ between alternatives $A_{k}$ and $A_{i}$ for $\mathrm{DM} D_{p}(p=1,2, \ldots, q)$ is defined to measure the inconsistency degree between the objective ranking determined by $R_{k}^{p}$ and $R_{i}^{p}$ and the subjective preference $(k, i) \in \Omega^{p}$, given by DM $D_{p}(p=1,2, \ldots, q)$ as

$$
\theta_{k i}^{p}= \begin{cases}\hat{h}_{S}^{p}(k, i)\left(R_{i}^{p}-R_{k}^{p}\right), & R_{k}^{p}<R_{i}^{p} \\ 0, & R_{k}^{p} \geq R_{i}^{p} \quad, p=1,2, \ldots, q ; k, i=1,2, \ldots, m .\end{cases}
$$

For convenience, we rewrite the inconsistency index as $\theta_{k i}^{p}=\hat{h}_{S}^{p}(k, i) \max \left\{0, R_{i}^{p}-R_{k}^{p}\right\}$. Hence, the hesitant fuzzy 2-dimension linguistic group inconsistency index can be calculated as follows:

$$
\theta=\sum_{p=1}^{q} \sum_{(k, i) \in \Omega^{p}} \theta_{k i}^{p}=\sum_{p=1}^{q} \sum_{(k, i) \in \Omega^{p}}\left(\hat{h}_{S}^{p}(k, i) \max \left\{0, R_{i}^{p}-R_{k}^{p}\right\}\right) .
$$

Analogously, the consistency index $\psi_{k i}^{p}$ between alternatives $A_{k}$ and $A_{i}$ for DM $D_{p}(p=$ $1,2, \ldots, q)$ is defined to measure the consistency degree between the objective ranking determined by $R_{k}^{p}$ and $R_{i}^{p}$ and the subjective preference $(k, i) \in \Omega^{p}$, given by $\operatorname{DM} D_{p}(p=$ $1,2, \ldots, q)$ as

$$
\psi_{k i}^{p}=\left\{\begin{array}{ll}
\hat{h}_{S}^{p}(k, i)\left(R_{k}^{p}-R_{i}^{p}\right), & R_{k}^{p} \geq R_{i}^{p} \\
0, & R_{k}^{p}<R_{i}^{p}
\end{array}, p=1,2, \ldots, q ; k, i=1,2, \ldots, m .\right.
$$

Furthermore, Equation (17) is rewritten as $\psi_{k i}^{p}=\hat{h}_{S}^{p}(k, i) \max \left\{0, R_{k}^{p}-R_{i}^{p}\right\}$. Hence, the hesitant fuzzy 2-dimension linguistic group consistency index can be calculated as

$$
\psi=\sum_{p=1}^{q} \sum_{(k, i) \in \Omega^{p}} \psi_{k i}^{p}=\sum_{p=1}^{q} \sum_{(k, i) \in \Omega^{p}}\left(\hat{h}_{S}^{p}(k, i) \max \left\{0, R_{k}^{p}-R_{i}^{p}\right\}\right) .
$$

Remark 1. There exist fuzziness and hesitation for DMs when making pairwise comparisons of alternatives. Since HF2DLTS is more flexible and comprehensive in representing hesitant qualitative preference information, this paper first introduces hesitant fuzzy 2-dimension linguistic truth degrees to describe the pairwise comparisons between alternatives. Due to the truth degrees 
$\hat{h}_{S}^{p}(k, i)$ denoted by HF2DLEs, the constructed group inconsistency and consistency indices defined in this paper are all HF2DLEs.

\subsection{Bi-Objective Hesitant Fuzzy 2-Dimension Linguistic Programming Model}

Since the group inconsistency and consistency indices reflect the overall inconsistency and consistency between the objective rankings and subjective preferences, the smaller the group inconsistency index and the larger the group consistency index, the better the model characterizes the DMs' decision rationales. Driven by this idea, a bi-objective hesitant fuzzy 2-dimension linguistic programming model is constructed to determine the criteria weights, HF2DLPIS and HF2DLNIS, as follows:

$$
\begin{aligned}
& \min \{\theta\}, \max \{\psi\}, \\
& \text { s.t. }\left\{\begin{array}{c}
\psi-\theta \geq \hat{h} \\
w \in \Lambda
\end{array}\right.
\end{aligned}
$$

where the objective functions involve minimizing the group inconsistency index $\theta$ and maximizing the group consistency index $\psi ; \hat{h}$ is a hesitant fuzzy 2-dimension linguistic threshold given by DMs in advance to ensure that the group consistency is not smaller than the group inconsistency; $w=\left(w_{1}, w_{2}, \ldots, w_{n}\right)^{T}$ is the weight vector of criteria; and $\Lambda$ is the incomplete preference information on criteria. In terms of Equations (16) and (18), it yields that

$$
\psi-\theta=\sum_{p=1}^{q} \sum_{(k, i) \in \Omega^{p}}\left(\hat{h}_{S}^{p}(k, i)\left(R_{k}^{p}-R_{i}^{p}\right)\right) .
$$

For each $(k, i) \in \Omega^{p}$, let $\lambda_{k i}^{p}=\max \left\{0, R_{i}^{p}-R_{k}^{p}\right\}$ and $\tau_{k i}^{p}=\max \left\{0, R_{k}^{p}-R_{i}^{p}\right\}$, then $\lambda_{k i}^{p} \geq 0, \tau_{k i}^{p} \geq 0, \lambda_{k i}^{p} \geq R_{i}^{p}-R_{k}^{p}$ and $\tau_{k i}^{p} \geq R_{k}^{p}-R_{i}^{p}$. Thus, Equation (19) can be rewritten as follows:

$$
\begin{aligned}
& \min \left\{\theta=\sum_{p=1}^{q} \sum_{(k, i) \in \Omega^{p}}\left(\lambda_{k i}^{p} \hat{h}_{S}^{p}(k, i)\right)\right\}, \\
& \max \left\{\psi=\sum_{p=1}^{q} \sum_{(k, i) \in \Omega^{p}}\left(\tau_{k i}^{p} \hat{h}_{S}^{p}(k, i)\right)\right\}, \\
& \text { s.t. }\left\{\begin{array}{l}
\sum_{p=1}^{q} \sum_{(k, i) \in \Omega^{p}}\left(\hat{h}_{S}^{p}(k, i)\left(R_{k}^{p}-R_{i}^{p}\right)\right) \geq \hat{h} \\
R_{k}^{p}-R_{i}^{p}+\lambda_{k i}^{p} \geq 0,(k, i) \in \Omega^{p} ; p=1,2, \ldots, q \\
R_{i}^{p}-R_{k}^{p}+\tau_{k i}^{p} \geq 0,(k, i) \in \Omega^{p} ; p=1,2, \ldots, q \\
\lambda_{k i}^{p} \geq 0, \tau_{k i}^{p} \geq 0,(k, i) \in \Omega^{p} ; p=1,2, \ldots, q \\
w \in \Lambda
\end{array}\right.
\end{aligned}
$$

Let $T_{k i}^{p}=R_{k}^{p}-R_{i}^{p}$; then, using Equation (14), we have

$$
\begin{aligned}
T_{k i}^{p} & =\frac{d_{k}^{p-}}{d_{k}^{p+}+d_{k}^{p-}}-\frac{d_{i}^{p-}}{d_{i}^{p+}+d_{i}^{p-}}=\frac{\sum_{j=1}^{n} w_{j} \sum_{l=1}^{L} \frac{1}{L}\left(\eta_{k j}^{(l)}-\eta_{j-}^{(l)}\right)^{2}}{\sum_{j=1}^{n} w_{j} \sum_{l=1}^{L} \frac{1}{L}\left(\eta_{k j}^{(l)}-\eta_{j+}^{(l)}\right)^{2}+\sum_{j=1}^{n} w_{j} \sum_{l=1}^{L} \frac{1}{L}\left(\eta_{k j}^{(l)}-\eta_{j-}^{(l)}\right)^{2}} \\
& -\frac{\sum_{j=1}^{n} w_{j} \sum_{l=1}^{L} \frac{1}{L}\left(\eta_{i j}^{(l)}-\eta_{j-}^{(l)}\right)^{2}}{\sum_{j=1}^{n} w_{j} \sum_{l=1}^{L} \frac{1}{L}\left(\eta_{i j}^{(l)}-\eta_{j+}^{(l)}\right)^{2}+\sum_{j=1}^{n} w_{j} \sum_{l=1}^{L} \frac{1}{L}\left(\eta_{i j}^{(l)}-\eta_{j-}^{(l)}\right)^{2}}, p=1,2, \ldots, q ; k, i=1,2, \ldots, m .
\end{aligned}
$$

where $\eta_{k j}^{(l)}$ is the $l$-th smallest element of $\left\{f\left(\dot{s}_{a_{k j}}^{(p)}\right) \cdot f\left(\ddot{s}_{b_{k j}}^{(p)}\right),\left(\dot{s}_{a_{k j}}^{(p)}, \ddot{s}_{b_{k j}}^{(p)}\right) \mid \hat{h}_{S_{k j}}^{p}\right\}$. 
Hence, Equation (21) can be further written as the following bi-objective hesitant fuzzy 2-dimension linguistic programming model:

$$
\begin{aligned}
& \min \left\{\theta=\sum_{p=1}^{q} \sum_{(k, i) \in \Omega^{p}}\left(\lambda_{k i}^{p} \hat{h}_{S}^{p}(k, i)\right)\right\}, \\
& \max \left\{\psi=\sum_{p=1}^{q} \sum_{(k, i) \in \Omega^{p}}\left(\tau_{k i}^{p} \hat{h}_{S}^{p}(k, i)\right)\right\}, \\
& \text { s.t. }\left\{\begin{array}{l}
\sum_{p=1}^{q} \sum_{(k, i) \in \Omega^{p}}\left(T_{k i}^{p} \hat{h}_{S}^{p}(k, i)\right) \geq \hat{h} \\
T_{k i}^{p}+\lambda_{k i}^{p} \geq 0,(k, i) \in \Omega^{p} ; p=1,2, \ldots, q \\
\tau_{k i}^{p}-T_{k i}^{p} \geq 0,(k, i) \in \Omega^{p} ; p=1,2, \ldots, q \\
\lambda_{k i}^{p} \geq 0, \tau_{k i}^{p} \geq 0,(k, i) \in \Omega^{p} ; p=1,2, \ldots, q \\
w \in \Lambda
\end{array}\right.
\end{aligned}
$$

Remark 2. In Equation (23), there exist $2 \sum_{p=1}^{q}\left|\Omega^{p}\right|+n+2(L \times n)$ unknown variables that need to be determined, i.e., $2 \sum_{p=1}^{q}\left|\Omega^{p}\right|$ variables $\lambda_{k i}^{p}$ and $\tau_{k i}^{p}\left((k, i) \in \Omega^{p}\right) ; n$ criteria weights $w_{j}(j=1,2, \ldots, n) ; L \times n$ variables of HF2DLPIS; $L \times n$ variables of HF2DLNIS; $3 \sum_{p=1}^{q}\left|\Omega^{p}\right|+1$ equalities; $2 \sum_{p=1}^{q}\left|\Omega^{p}\right|+1$ inequalities (excluding the non-negative constraints for variables and $\Lambda$ ). To determine the unknown variables, the number of $2 \sum_{p=1}^{q}\left|\Omega^{p}\right|+1$ inequalities should not be too small. In general, the larger $\left|\Omega^{p}\right|$ (pairwise comparisons between alternatives), the more precise and reliable determining the weight vectors of criteria w, HF2DLPIS $\hat{h}_{S}^{+}$and HF2DLNIS $\hat{h}_{S}^{-}$.

\subsection{Solution for Bi-Objective Hesitant Fuzzy 2-Dimension Linguistic Programming Model}

Since both objective functions and constraints of Equation (23) contain HF2DLEs $\hat{h}_{S}^{p}(k, i)\left((k, i) \in \Omega^{p}\right)$ and $\hat{h}$, we thus have to develop an effective method to solve the constructed bi-objective hesitant fuzzy 2-dimension linguistic programming model.

According to the operational laws of HF2DLEs, two objective functions of Equation (23) are HF2DLEs, which are expressed as follows:

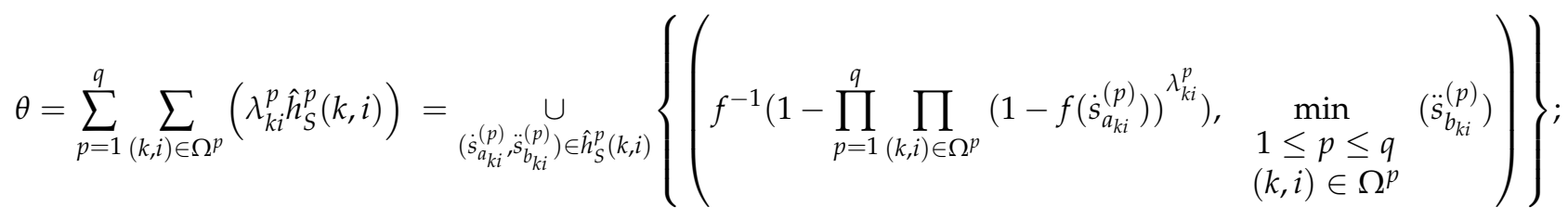

$$
\begin{aligned}
& \psi=\sum_{p=1}^{q} \sum_{(k, i) \in \Omega^{p}}\left(\tau_{k i}^{p} \hat{h}_{S}^{p}(k, i)\right)=\underset{\left(\dot{s}_{a_{k i}}^{(p)} \dot{s}_{k i}^{(p)}\right) \in \hat{h}_{S}^{p}(k, i)}{\cup}\left\{\left(f^{-1}\left(1-\prod_{p=1}^{q} \prod_{(k, i) \in \Omega^{p}}\left(1-f\left(\dot{s}_{a_{k i}}^{(p)}\right)\right)^{\left.\tau_{k i}^{p}\right)} \min _{\substack{1 \leq p \leq q \\
(k, i) \in \Omega^{p}}}\left(\ddot{s}_{b_{k i}}^{(p)}\right)\right)\right\} .\right.
\end{aligned}
$$

Additionally, the left hand of the first constraint in Equation (23) is also an HF2DLE, which is expressed as follows: 


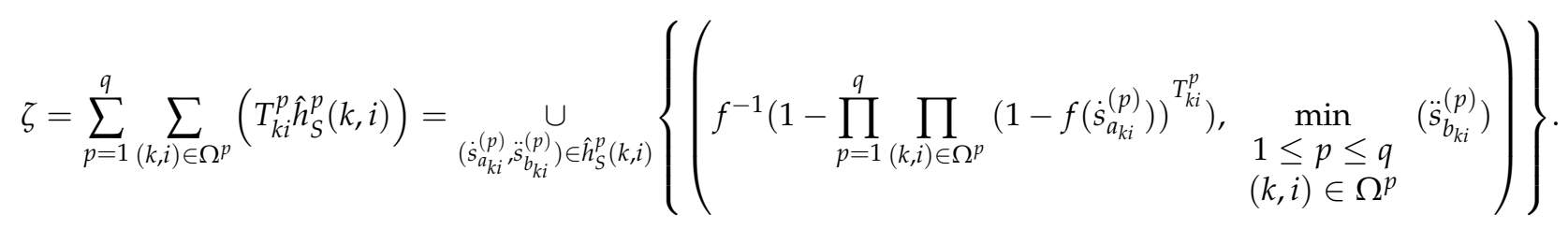

Based on Equation (9), the score values of $\theta, \psi$ and $\zeta$ are obtained as

$$
\begin{aligned}
& \left.E(\theta)=\frac{1}{\# \theta} \sum_{\substack{\left(\dot{s}_{a_{k i}}^{(p)} \dot{s}_{b_{k i}}^{(p)}\right) \in \hat{h}_{S}^{p}(k, i)\\
}}\left(1-\prod_{p=1}^{q} \prod_{(k, i) \in \Omega^{p}}\left(1-f\left(\dot{s}_{a_{k i}}^{(p)}\right)\right)^{\lambda_{k i}^{p}}\right) \cdot f\left(\min _{\substack{1 \leq p \leq q \\
(k, i) \in \Omega^{p}}}\left(\ddot{s}_{b_{k i}}^{(p)}\right)\right)\right), \\
& E(\psi)=\frac{1}{\# \psi} \sum_{\left(\dot{s}_{a_{k i}}^{(p)} \dot{s}_{b_{k i}}^{(p)}\right) \in \hat{h}_{S}^{p}(k, i)}\left(\left(1-\prod_{p=1}^{q} \prod_{(k, i) \in \Omega^{p}}\left(1-f\left(\dot{s}_{a_{k i}}^{(p)}\right)\right)^{\tau_{k i}^{p}}\right) \cdot f\left(\min _{\substack{1 \leq p \leq q \\
(k, i) \in \Omega^{p}}}\left(\ddot{s}_{b_{k i}}^{(p)}\right)\right)\right), \\
& E(\zeta)=\frac{1}{\# \zeta} \sum_{\left(\dot{s}_{a_{k i}}^{(p)},\left(\dot{s}_{b_{k i}}^{(p)}\right) \hat{h}_{S}^{p}(k, i)\right.}\left(\left(1-\prod_{p=1}^{q} \prod_{(k, i) \in \Omega^{p}}\left(1-f\left(\dot{s}_{a_{k i}}^{(p)}\right)\right)^{T_{k i}^{p}}\right) \cdot f\left(\min _{\substack{1 \leq p \leq q \\
(k, i) \in \Omega^{p}}}\left(\ddot{s}_{b_{k i}}^{(p)}\right)\right)\right) \text {, }
\end{aligned}
$$

where $\# \theta, \# \psi$ and $\# \zeta$ are the numbers of elements in $\theta, \psi$ and $\zeta$.

Based on score values, the first objective function $\min \{\theta\}$ of Equation (23) is equivalent to minimizing the corresponding score value $\min \{E(\theta)\}$; the second objective function $\max \{\psi\}$ of Equation (23) is equivalent to maximizing the corresponding score value $\max \{E(\psi)\}$. The first constraint of Equation (23) is equivalent to $E(\zeta) \geq E(\hat{h})$. Therefore, based on the linear weighted sum method, Equation (23) is transformed into the following single-objective programming model:

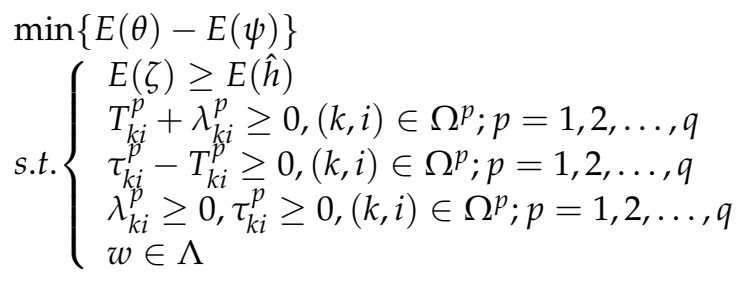

Though Equation (30) is non-linear programming, it can be solved using the usual software, such as Lingo and MATLAB. By solving Equation (30), the weight vector of criteria $w=\left(w_{1}, w_{2}, \ldots, w_{n}\right)^{T}$, HF2DLPIS $\hat{h}_{S}^{+}=\left(\widehat{h}_{S_{1}}^{+}, \widehat{h}_{S_{2}}^{+}, \ldots, \widehat{h}_{S_{n}}^{+}\right)$and HF2DLNIS $\hat{h}_{S}^{-}=\left(\widehat{h}_{S_{1}}^{-}, \widehat{h}_{S_{2}}^{-}, \ldots, \widehat{h}_{S_{n}}^{-}\right)$can be obtained.

\subsection{A Novel Fuzzy Programming Method for Hesitant Fuzzy 2-Dimension Linguistic MCGDM}

On the basis of the above analysis, a novel fuzzy programming method is developed for solving MCGDM problems, in which the evaluation values of alternatives and the truth degrees of pairwise comparisons between alternatives are expressed as HF2DLEs, and the preferences over alternatives and criteria are incomplete. The proposed method involves the following concrete steps:

Step 1. Identify the evaluation criteria $C_{j}(j=1,2, \ldots, n)$ and all feasible alternatives $A_{i}(i=1,2, \ldots, m)$ and form the set of $\mathrm{DMs}_{p}(p=1,2, \ldots, q)$; 
Step 2. Elicit the individual hesitant fuzzy 2-dimension linguistic decision matrices $\hat{H}_{S}^{(p)}=\left(\hat{h}_{S_{i j}}^{(p)}\right)_{m \times n}(p=1,2, \ldots, q)$, the incomplete weight information $\Lambda$, and the preference relations on alternatives $\Omega^{p}=\left\{<(k, i), \hat{h}_{S}^{p}(k, i)>\mid A_{k} \succeq_{p} A_{i}\right\}(p=1,2, \ldots, q)$;

Step 3. Construct the bi-objective hesitant fuzzy 2-dimension linguistic programming model according to Equation (23) and then transform it into a single objective programming model as Equation (30);

Step 4. Solve Equation (30) to derive the weight vector of criteria $w=\left(w_{1}, w_{2}, \ldots, w_{n}\right)^{T}$, HF2DLPIS $\hat{h}_{S}^{+}=\left(\widehat{h}_{S_{1}}^{+}, \widehat{h}_{S_{2}}^{+}, \ldots, \widehat{h}_{S_{n}}^{+}\right)$, and HF2DLNIS $\hat{h}_{S}^{-}=\left(\widehat{h}_{S_{1}}^{-}, \widehat{h}_{S_{2}}^{-}, \ldots, \widehat{h}_{S_{n}}^{-}\right)$;

Step 5. Calculate the RCD $R_{i}^{p}$ of the alternative $A_{i}(i=1,2, \ldots, m)$ to HF2DLPIS $\hat{h}_{S}^{+}$for the $\mathrm{DM} D_{p}(p=1,2, \ldots, q)$ by Equation (14);

Step 6. Rank all feasible alternatives for the DM $D_{p}(p=1,2, \ldots, q)$ according to the descending order of RCDs $R_{i}^{p}(p=1,2, \ldots, q ; i=1,2, \ldots, m)$ and generate the individual rankings of alternatives;

Step 7. Construct the individual ranking matrix $X^{p}=\left(x_{i j}^{p}\right)_{m \times m}$ for the DM $D_{p}(p=$ $1,2, \ldots, q)$, where

$$
x_{i j}^{p}=\left\{\begin{array}{l}
1, D_{p} \text { ranks } A_{j} \text { in the } i \text {-th position } \\
0, \text { otherwise }
\end{array}, p=1,2, \ldots, q ; i, j=1,2, \ldots, m,\right.
$$

This means that if the $\operatorname{DM} D_{p}(p=1,2, \ldots, q)$ ranks alternative $A_{j}(j=1,2, \ldots, m)$ in the $i$-th position $i \in\{1,2, \ldots, m\}$, then $x_{i j}^{p}=1$; otherwise, $x_{i j}^{p}=0$;

Step 8. Determine the group ranking matrix $X=\left(x_{i j}\right)_{m \times m}$ of all alternatives and select the most desirable alternative.

Suppose that the group ranks alternative $A_{j}(j=1,2, \ldots, m)$ in the $i$-th position $i \in\{1,2, \ldots, m\}$, i.e.,

$$
x_{i j}=\left\{\begin{array}{l}
1, \text { group ranks } A_{j} \text { in the } i \text {-th position } \\
0, \text { otherwise }
\end{array}, i, j=1,2, \ldots, m,\right.
$$

which needs to be determined.

Generally, the individual ranking of alternatives for each DM should be as close as possible to the group ranking. Thus, to determine the group ranking of all feasible alternatives, a single-objective assignment model is constructed as follows:

$$
\begin{aligned}
& \min \sum_{p=1}^{q} \sum_{i=1}^{m} \sum_{j=1}^{m}\left|x_{i j}^{p}-x_{i j}\right| \\
& \text { s.t. }\left\{\begin{array}{l}
\sum_{i=1}^{m} x_{i j}=1, j=1,2, \ldots, m \\
\sum_{j=1}^{m} x_{i j}=1, i=1,2, \ldots, m \\
x_{i j}=0 \text { or } 1, i, j=1,2, \ldots, m
\end{array}\right.
\end{aligned}
$$

where the objective function aims to minimize the deviations between individual rankings and group ranking; the first constraint ensures that one alternative is only in one position; the second constraint ensures that one position only has one alternative. By solving Equation (33), the group ranking of all feasible alternatives is obtained, and the most desirable alternative can be determined.

\section{Case Study}

In this section, a case study about energy policy selection adopted from ref. [25] is provided to illustrate the feasibility of the proposed method. Further, the effectiveness of the proposed method is demonstrated by comparing it with the existing methods. 


\subsection{Energy Policy Selection}

As a country with great energy consumption, China relies on coal as its main energy source. However, with the increasing contradiction between economic development and environmental pollution, air pollution and other environmental hazards caused by coal consumption are becoming increasingly severe, and sustainable development in terms of social aspects, energy, and the environment is facing great challenges. Therefore, the energy sector urgently needs to switch to low carbon, environmentally friendly, and clean resources.

In recent years, the Chinese government has issued a series of energy policies on energy conservation, emission reduction, and environmental protection, resulting in a decline in the proportion of coal consumption in China's energy structure year by year, while the proportion of clean energy such as natural gas has gradually increased. From 2016 to 2019, the proportion of raw coal in China's total energy consumption has decreased from $62 \%$ to $57.7 \%$, and the proportion of natural gas has increased from $6.4 \%$ to $8.1 \%$, which reflects the trend of continuous optimization of China's energy structure.

The selection of the best energy policy plays an important role in energy investment and policy development. For evaluating energy policy, there are many criteria that should be taken into consideration, such as economic $\left(C_{1}\right)$, technological $\left(C_{2}\right)$, and environmental analyses $\left(C_{3}\right)$, as well as social-political impact $\left(C_{4}\right)$. Energy policies (alternatives) of this study were selected as biomass power plants $\left(A_{1}\right)$, nuclear energy $\left(A_{2}\right)$, hydropower $\left(A_{3}\right)$, wind power $\left(A_{4}\right)$, and solar energy $\left(A_{5}\right)$. To collect the decision data, the expert interview method was used to invite a committee of three DMs $D_{p}(p=1,2,3)$ to evaluate the performances of the five alternatives in terms of these four criteria anonymously, in which the DMs may hesitate among several linguistic terms, and the reliability of each term was different. To comprehensively preserve the preference information, the hesitant linguistic information given by DMs was transformed into HF2DLEs based on the first-dimension LTS $S^{(1)}=\left\{\dot{s}_{0}=\right.$ extremely poor, $\dot{s}_{1}=$ very poor, $\dot{s}_{2}=$ poor, $\dot{s}_{3}=$ medium, $\dot{s}_{4}=$ good, $\dot{s}_{5}=$ very good, $\dot{s}_{6}=$ extremely good $\}$ and the second dimension LTS $S^{(2)}=\left\{\ddot{s}_{0}=\right.$ extremely low certainty, $\ddot{s}_{1}=$ very low certainty, $\ddot{s}_{2}=$ low certainty, $\ddot{s}_{3}=$ certainty, $\ddot{s}_{4}=$ high certainty, $\ddot{s}_{5}=$ very high certainty, $\ddot{s}_{6}=$ extremely high certainty\}. For example, DM $D_{1}$ provided their evaluation of alternative $A_{1}$ on criterion $C_{1}$ as "I am highly certain it is good, but I have low certainty it is very good", and thus, an HF2DLE was determined as $\left\{\left(\dot{s}_{4}, \ddot{s}_{4}\right),\left(\dot{s}_{5}, \ddot{s}_{2}\right)\right\}$. Hereby, three individual hesitant fuzzy 2-dimension linguistic decision matrices $\hat{H}_{S}^{(p)}=\left(\hat{h}_{S_{i j}}^{(p)}\right)_{5 \times 4}$ $(p=1,2,3)$ were obtained, which are shown in Table 1.

Table 1. Individual hesitant fuzzy 2-dimension linguistic decision matrices.

\begin{tabular}{|c|c|c|c|c|c|}
\hline DMs & Alternatives & $C_{1}$ & $C_{2}$ & $C_{3}$ & $C_{4}$ \\
\hline$D_{1}$ & $\begin{array}{l}A_{1} \\
A_{2} \\
A_{3} \\
A_{4} \\
A_{5}\end{array}$ & $\begin{array}{l}\left\{\left(\dot{s}_{4}, \ddot{s}_{4}\right),\left(\dot{s}_{5}, \ddot{s}_{2}\right)\right\} \\
\left.\left(\dot{s}_{1}, \ddot{s}_{5}\right),\left(\dot{s}_{2}, \ddot{s}_{3}\right)\right\} \\
\left\{\left(\dot{s}_{2}, \ddot{s}_{1}\right),\left(\dot{s}_{3}, \ddot{s}_{4}\right)\right\} \\
\left\{\left(\dot{s}_{4}, \ddot{s}_{5}\right)\right\} \\
\left\{\left(\dot{s}_{5}, \ddot{s}_{4}\right),\left(\dot{s}_{6}, \ddot{s}_{1}\right)\right\}\end{array}$ & 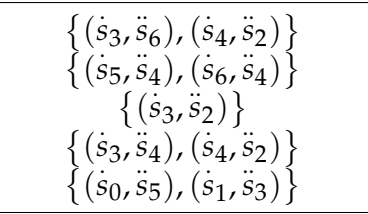 & 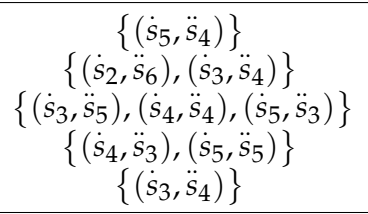 & $\begin{array}{c}\left\{\left(\dot{s}_{2}, \ddot{s}_{4}\right),\left(\dot{s}_{3}, \ddot{s}_{5}\right),\left(\dot{s}_{4}, \ddot{s}_{2}\right)\right\} \\
\left\{\left(\dot{s}_{3}, \ddot{s}_{3}\right),\left(\dot{s}_{4}, \ddot{s}_{5}\right)\right\} \\
\quad\left\{\left(\dot{s}_{4}, \ddot{s}_{5}\right)\right\} \\
\left\{\left(\dot{s}_{1}, \ddot{s}_{4}\right),\left(\dot{s}_{2}, \ddot{s}_{4}\right),\left(\dot{s}_{3}, \ddot{s}_{2}\right)\right\} \\
\left\{\left(\dot{s}_{1}, \ddot{s}_{4}\right),\left(\dot{s}_{2}, \ddot{s}_{3}\right)\right\}\end{array}$ \\
\hline$D_{2}$ & $\begin{array}{l}A_{1} \\
A_{2} \\
A_{3} \\
A_{4} \\
A_{5}\end{array}$ & 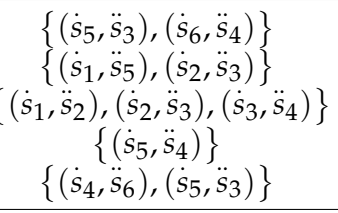 & $\begin{array}{c}\left\{\left(\dot{s}_{5}, \ddot{s}_{4}\right)\right\} \\
\left\{\left(\dot{s}_{4}, \ddot{s}_{5}\right),\left(\dot{s}_{5}, \ddot{s}_{3}\right),\left(\dot{s}_{6}, \ddot{s}_{4}\right)\right\} \\
\left\{\left(\dot{s}_{5}, \ddot{s}_{5}\right)\right\} \\
\left\{\left(\dot{s}_{3}, \ddot{s}_{5}\right),\left(\dot{s}_{4}, \ddot{s}_{2}\right)\right\} \\
\left\{\left(\dot{s}_{5}, \ddot{s}_{4}\right),\left(\dot{s}_{6}, \ddot{s}_{3}\right)\right\}\end{array}$ & $\begin{array}{c}\left\{\left(\dot{s}_{3}, \ddot{s}_{4}\right),\left(\dot{s}_{4}, \ddot{s}_{3}\right)\right\} \\
\left\{\left(\dot{s}_{5}, \dot{s}_{4}\right),\left(\dot{s}_{6}, \dot{s}_{3}\right)\right\} \\
\left\{\left(\dot{s}_{4}, \ddot{s}_{4}\right),\left(\dot{s}_{5}, \ddot{s}_{6}\right)\right\} \\
\left\{\left(\dot{s}_{5}, \ddot{s}_{3}\right)\right\} \\
\left\{\left(\dot{s}_{3}, \ddot{s}_{2}\right),\left(\dot{s}_{4}, \ddot{s}_{4}\right)\right\}\end{array}$ & $\begin{array}{c}\left\{\left(\dot{s}_{4}, \ddot{s}_{6}\right),\left(\dot{s}_{5}, \ddot{s}_{3}\right)\right\} \\
\left\{\left(\dot{s}_{5}, \ddot{s}_{3}\right)\right\} \\
\left\{\left(\dot{s}_{1}, \ddot{s}_{4}\right),\left(\dot{s}_{2}, \ddot{s}_{2}\right)\right\} \\
\left\{\left(\dot{s}_{3}, \ddot{s}_{5}\right),\left(\dot{s}_{4}, \ddot{s}_{3}\right)\right\} \\
\left\{\left(\dot{s}_{3}, \ddot{s}_{4}\right)\right\}\end{array}$ \\
\hline$D_{3}$ & $\begin{array}{l}A_{1} \\
A_{2} \\
A_{3} \\
A_{4} \\
A_{5} \\
\end{array}$ & $\begin{array}{l}\left\{\left(\dot{s}_{4}, \ddot{s}_{6}\right),\left(\dot{s}_{5}, \ddot{s}_{2}\right)\right\} \\
\left\{\left(\dot{s}_{5}, \ddot{s}_{5}\right)\right\} \\
\left\{\left(\dot{s}_{3}, \ddot{s}_{5}\right),\left(\dot{s}_{4}, \ddot{s}_{1}\right)\right\} \\
\left\{\left(\dot{s}_{2}, \ddot{s}_{4}\right),\left(\dot{s}_{3}, \ddot{s}_{1}\right)\right\} \\
\left\{\left(\dot{s}_{4}, \ddot{s}_{3}\right)\right\}\end{array}$ & $\begin{array}{l}\left\{\left(\dot{s}_{2}, \ddot{s}_{3}\right),\left(\dot{s}_{3}, \ddot{s}_{4}\right),\left(\dot{s}_{4}, \ddot{s}_{1}\right)\right\} \\
\left\{\left(\dot{s}_{5}, \ddot{s}_{4}\right),\left(\dot{s}_{6}, \dot{s}_{3}\right)\right\} \\
\left\{\left(\dot{s}_{5}, \ddot{s}_{3}\right)\right\} \\
\left\{\left(\dot{s}_{0}, \ddot{s}_{5}\right),\left(\dot{s}_{1}, \ddot{s}_{2}\right)\right\} \\
\left\{\left(\dot{s}_{4}, \ddot{s}_{6}\right),\left(\dot{s}_{5}, \ddot{s}_{3}\right)\right\}\end{array}$ & $\begin{array}{c}\left\{\left(\dot{s}_{3}, \ddot{s}_{4}\right)\right\} \\
\left\{\left(\dot{s}_{1}, \ddot{s}_{3}\right),\left(\dot{s}_{2}, \ddot{s}_{5}\right)\right\} \\
\left\{\left(\dot{s}_{2}, \ddot{s}_{5}\right),\left(\dot{s}_{3}, \ddot{s}_{1}\right)\right\} \\
\left\{\left(\dot{s}_{1}, \ddot{s}_{3}\right),\left(\dot{s}_{2}, \ddot{s}_{4}\right),\left(\dot{s}_{3}, \ddot{s}_{2}\right)\right\} \\
\left\{\left(\dot{s}_{4}, \ddot{s}_{0}\right),\left(\dot{s}_{5}, \ddot{s}_{3}\right),\left(\dot{s}_{6}, \ddot{s}_{2}\right)\right\}\end{array}$ & $\begin{array}{l}\left\{\left(\dot{s}_{4}, \ddot{s}_{5}\right)\right\} \\
\left\{\left(\dot{s}_{3}, \ddot{s}_{5}\right),\left(\dot{s}_{4}, \ddot{s}_{6}\right)\right\} \\
\left\{\left(\dot{s}_{4}, \ddot{s}_{0}\right),\left(\dot{s}_{5}, \ddot{s}_{3}\right)\right\} \\
\quad\left\{\left(\dot{s}_{1}, \ddot{s}_{5}\right)\right\} \\
\left\{\left(\dot{s}_{2}, \ddot{s}_{5}\right)\right\}\end{array}$ \\
\hline
\end{tabular}


Meanwhile, the DMs also provided the incomplete weight information of criteria as $\Lambda=\left\{\mu \in \Lambda \mid 0.1 \leq w_{1}-w_{2} \leq 0.25, w_{2} \geq 2 w_{3}, 0.4 \leq w_{2} \leq 0.6\right\}$ and their incomplete preference relations of pairwise comparisons between alternatives as follows:

$$
\begin{aligned}
& \Omega^{1}=\left\{<(2,1), \hat{h}_{S}^{1}(2,1)>,<(1,3), \hat{h}_{S}^{1}(1,3)>,<(5,4), \hat{h}_{S}^{1}(5,4)>,<(2,5), \hat{h}_{S}^{1}(2,5)>,\right. \\
&\left.<(3,2), \hat{h}_{S}^{1}(3,2)>,<(3,4), \hat{h}_{S}^{1}(3,4)>\right\}, \\
& \Omega^{2}=\left\{<(1,2), \hat{h}_{S}^{2}(1,2)>,<(3,1), \hat{h}_{S}^{2}(3,1)>,<(4,5), \hat{h}_{S}^{2}(4,5)>,<(5,2), \hat{h}_{S}^{2}(5,2)>,\right. \\
&\left.<(2,3), \hat{h}_{S}^{2}(2,3)>,<(4,3), \hat{h}_{S}^{2}(4,3)>\right\}, \\
& \Omega^{3}=\left\{<(2,4), \hat{h}_{S}^{3}(2,4)>,<(1,4), \hat{h}_{S}^{3}(1,4)>,<(5,1), \hat{h}_{S}^{3}(5,1)>,<(5,3), \hat{h}_{S}^{3}(5,3)>\right\},
\end{aligned}
$$

where the corresponding hesitant fuzzy 2-dimension linguistic truth degrees are specified as

$$
\begin{gathered}
\hat{h}_{S}^{1}(2,1)=\left\{\left(\dot{s}_{1}, \ddot{s}_{4}\right),\left(\dot{s}_{2}, \ddot{s}_{5}\right)\right\}, \hat{h}_{S}^{1}(1,3)=\left\{\left(\dot{s}_{2}, \ddot{s}_{5}\right)\right\}, \hat{h}_{S}^{1}(5,4)=\left\{\left(\dot{s}_{2}, \ddot{s}_{3}\right),\left(\dot{s}_{3}, \ddot{s}_{4}\right),\left(\dot{s}_{4}, \ddot{s}_{5}\right)\right\}, \\
\hat{h}_{S}^{1}(2,5)=\left\{\left(\dot{s}_{0}, \ddot{s}_{4}\right),\left(\dot{s}_{1}, \ddot{s}_{5}\right)\right\}, \hat{h}_{S}^{1}(3,2)=\left\{\left(\dot{s}_{4}, \ddot{s}_{3}\right),\left(\dot{s}_{5}, \ddot{s}_{4}\right)\right\}, \hat{h}_{S}^{1}(3,4)=\left\{\left(\dot{s}_{2}, \ddot{s}_{3}\right),\left(\dot{s}_{3}, \ddot{s}_{4}\right)\right\}, \\
\hat{h}_{S}^{2}(1,2)=\left\{\left(\dot{s}_{2}, \ddot{s}_{5}\right),\left(\dot{s}_{3}, \ddot{s}_{6}\right)\right\}, \hat{h}_{S}^{2}(3,1)=\left\{\left(\dot{s}_{4}, \ddot{s}_{3}\right),\left(\dot{s}_{5}, \ddot{s}_{4}\right)\right\}, \hat{h}_{S}^{2}(4,5)=\left\{\left(\dot{s}_{0}, \ddot{s}_{4}\right),\left(\dot{s}_{1}, \ddot{s}_{5}\right),\left(\dot{s}_{2}, \ddot{s}_{6}\right)\right\}, \\
\hat{h}_{S}^{2}(5,2)=\left\{\left(\dot{s}_{0}, \ddot{s}_{6}\right)\right\}, \hat{h}_{S}^{2}(2,3)=\left\{\left(\dot{s}_{1}, \ddot{s}_{5}\right),\left(\dot{s}_{2}, \ddot{s}_{6}\right)\right\}, \hat{h}_{S}^{2}(4,3)=\left\{\left(\dot{s}_{2}, \ddot{s}_{4}\right),\left(\dot{s}_{3}, \ddot{s}_{5}\right)\right\}, \\
\hat{h}_{S}^{3}(2,4)=\left\{\left(\dot{s}_{2}, \ddot{s}_{6}\right),\left(\dot{s}_{3}, \ddot{s}_{6}\right)\right\}, \hat{h}_{S}^{3}(1,4)=\left\{\left(\dot{s}_{3}, \ddot{s}_{1}\right),\left(\dot{s}_{4}, \ddot{s}_{3}\right)\right\}, \hat{h}_{S}^{3}(5,1)=\left\{\left(\dot{s}_{1}, \ddot{s}_{3}\right)\right\}, \\
\hat{h}_{S}^{3}(5,3)=\left\{\left(\dot{s}_{0}, \ddot{s}_{5}\right),\left(\dot{s}_{1}, \ddot{s}_{6}\right)\right\} .
\end{gathered}
$$

In the following, we employ the proposed method in Section 3.4 to solve the above energy policy selection problem.

Since the criteria weights were incompletely known, and the HF2DLPIS and HF2DLNIS were unknown a priori, taking $\hat{h}=\left\{\left(\dot{s}_{0.01}, \ddot{s}_{0.01}\right)\right\}$, the bi-objective hesitant fuzzy 2dimension linguistic programming model can be constructed by Equation (23) to derive the criteria weights, HF2DLPIS and HF2DLNIS, and then to solve the constructed model effectively, it is transformed into the single-objective programming model according to Equation (30). By solving the single-objective programming model, the main components of the optimal solution can be obtained as follows:

(1) HF2DLPIS on each criterion:

$$
\begin{aligned}
& \hat{h}_{S_{1}}^{+}=\left\{\left(\dot{s}_{0.7686}, \ddot{s}_{2.4118}\right),\left(\dot{s}_{2.3206}, \ddot{s}_{0.7084}\right),\left(\dot{s}_{4.8912}, \ddot{s}_{4.2648}\right)\right\}, \\
& \hat{h}_{S_{2}}^{+}=\left\{\left(\dot{s}_{0.4292}, \ddot{s}_{1.1796}\right),\left(\dot{s}_{3.5970}, \ddot{s}_{4.2179}\right),\left(\dot{s}_{5.4019}, \ddot{s}_{5.1236}\right)\right\}, \\
& \hat{h}_{S_{3}}^{+}=\left\{\left(\dot{s}_{1.0177}, \ddot{s}_{2.7557}\right),\left(\dot{s}_{2.6634}, \ddot{s}_{2.2841}\right),\left(\dot{s}_{4.5686}, \ddot{s}_{2.5376}\right)\right\}, \\
& \hat{h}_{S_{4}}^{+}=\left\{\left(\dot{s}_{0.8396}, \ddot{s}_{2.4577}\right),\left(\dot{s}_{2.5270}, \ddot{s}_{1.8155}\right),\left(\dot{s}_{4.7413}, \ddot{s}_{3.5680}\right)\right\}
\end{aligned}
$$

(2) HF2DLNIS on each criterion:

$$
\begin{aligned}
& \hat{h}_{S_{1}}^{-}=\left\{\left(\dot{s}_{0.7130}, \ddot{s}_{1.5466}\right),\left(\dot{s}_{4.5015}, \ddot{s}_{5.2705}\right),\left(\dot{s}_{5.3104}, \ddot{s}_{2.9779}\right)\right\}, \\
& \hat{h}_{S_{2}}^{-}=\left\{\left(\dot{s}_{1.3569}, \ddot{s}_{2.7375}\right),\left(\dot{s}_{4.2017}, \ddot{s}_{5.0212}\right),\left(\dot{s}_{5.2284}, \ddot{s}_{3.7853}\right)\right\}, \\
& \hat{h}_{S_{3}}^{-}=\left\{\left(\dot{s}_{1.5449}, \ddot{s}_{3.2719}\right),\left(\dot{s}_{3.4415}, \ddot{s}_{3.8579}\right),\left(\dot{s}_{4.9604}, \ddot{s}_{3.5702}\right)\right\}, \\
& \hat{h}_{S_{4}}^{-}=\left\{\left(\dot{s}_{1.9102}, \ddot{s}_{3.5018}\right),\left(\dot{s}_{3.9837}, \ddot{s}_{4.6867}\right),\left(\dot{s}_{5.1090}, \ddot{s}_{3.3502}\right)\right\}
\end{aligned}
$$

(3) Weights of criteria:

$$
w_{1}=0.5453, w_{2}=0.4196, w_{3}=0.0094, w_{4}=0.0253
$$


Then, the distances between the alternative $A_{i}(i=1,2,3,4,5)$ and HF2DLPIS $\hat{h}_{S}^{+}$or HF2DLNIS $\hat{h}_{S}^{-}$can be calculated by Equations (12) and (13), as shown in Tables 2 and 3.

Table 2. Distances between alternatives and HF2DLPIS.

\begin{tabular}{cccccc}
\hline DMs & $\boldsymbol{A}_{\mathbf{1}}$ & $\boldsymbol{A}_{\mathbf{2}}$ & $\boldsymbol{A}_{\mathbf{3}}$ & $\boldsymbol{A}_{\mathbf{4}}$ & $\boldsymbol{A}_{\mathbf{5}}$ \\
\hline$D_{1}$ & 0.0903 & 0.0374 & 0.0879 & 0.0453 & 0.1702 \\
$D_{2}$ & 0.0607 & 0.0763 & 0.0415 & 0.0438 & 0.0884 \\
$D_{3}$ & 0.1523 & 0.0170 & 0.1080 & 0.1472 & 0.0383 \\
\hline
\end{tabular}

Table 3. Distances between alternatives and HF2DLNIS.

\begin{tabular}{cccccc}
\hline DMs & $A_{\mathbf{1}}$ & $\boldsymbol{A}_{\mathbf{2}}$ & $A_{\mathbf{3}}$ & $A_{\mathbf{4}}$ & $A_{\mathbf{5}}$ \\
\hline$D_{1}$ & 0.0320 & 0.0675 & 0.1414 & 0.1088 & 0.0986 \\
$D_{2}$ & 0.0704 & 0.0978 & 0.1003 & 0.1035 & 0.0028 \\
$D_{3}$ & 0.0445 & 0.0944 & 0.0832 & 0.1422 & 0.0867 \\
\hline
\end{tabular}

Using Equation (14), the RCD $R_{i}^{p}$ of alternative $A_{i}(i=1,2,3,4,5)$ to HF2DLPIS for DM $D_{p}(p=1,2,3)$ can be obtained, and the individual rankings of all alternatives are generated according to the descending order of RCDs, which are shown in Table 4.

Table 4. RCDs and individual rankings of alternatives.

\begin{tabular}{ccccccc}
\hline DMs & $\boldsymbol{A}_{\mathbf{1}}$ & $\boldsymbol{A}_{\mathbf{2}}$ & $\boldsymbol{A}_{\mathbf{3}}$ & $\boldsymbol{A}_{\mathbf{4}}$ & $\boldsymbol{A}_{\mathbf{5}}$ & Individual Rankings \\
\hline$D_{1}$ & 0.2613 & 0.6437 & 0.6165 & 0.7061 & 0.3669 & $A_{4} \succ A_{2} \succ A_{3} \succ A_{5} \succ A_{1}$ \\
$D_{2}$ & 0.5371 & 0.5617 & 0.7072 & 0.7026 & 0.0304 & $A_{3} \succ A_{4} \succ A_{2} \succ A_{1} \succ A_{5}$ \\
$D_{3}$ & 0.2262 & 0.8473 & 0.4353 & 0.4914 & 0.6933 & $A_{2} \succ A_{5} \succ A_{4} \succ A_{3} \succ A_{1}$ \\
\hline
\end{tabular}

Based on the individual rankings of alternatives, the individual ranking matrix $X^{p}=\left(x_{i j}^{p}\right)_{5 \times 5}$ for DM $D_{p}(p=1,2,3)$ is constructed by Equation (31) as follows:

$$
X^{1}=\left(\begin{array}{lllll}
0 & 0 & 0 & 1 & 0 \\
0 & 1 & 0 & 0 & 0 \\
0 & 0 & 1 & 0 & 0 \\
0 & 0 & 0 & 0 & 1 \\
1 & 0 & 0 & 0 & 0
\end{array}\right), X^{2}=\left(\begin{array}{lllll}
0 & 0 & 1 & 0 & 0 \\
0 & 0 & 0 & 1 & 0 \\
0 & 1 & 0 & 0 & 0 \\
1 & 0 & 0 & 0 & 0 \\
0 & 0 & 0 & 0 & 1
\end{array}\right), X^{3}=\left(\begin{array}{lllll}
0 & 1 & 0 & 0 & 0 \\
0 & 0 & 0 & 0 & 1 \\
0 & 0 & 0 & 1 & 0 \\
0 & 0 & 1 & 0 & 0 \\
1 & 0 & 0 & 0 & 0
\end{array}\right)
$$

According to Equation (33), the single-objective assignment model is constructed as

$$
\begin{aligned}
& \min \sum_{p=1}^{3} \sum_{i=1}^{5} \sum_{j=1}^{5}\left|x_{i j}^{p}-x_{i j}\right| \\
& \text { s.t. }\left\{\begin{array}{l}
\sum_{i=1}^{5} x_{i j}=1, j=1,2, \ldots, 5 \\
\sum_{j=1}^{5} x_{i j}=1, i=1,2, \ldots, 5 \\
x_{i j}=\text { oor } 1, i, j=1,2, \ldots, 5
\end{array}\right.
\end{aligned}
$$

By solving the above model, the group ranking matrix can be obtained, which is shown as follows:

$$
X=\left(\begin{array}{lllll}
0 & 0 & 1 & 0 & 0 \\
0 & 1 & 0 & 0 & 0 \\
0 & 0 & 0 & 1 & 0 \\
0 & 0 & 0 & 0 & 1 \\
1 & 0 & 0 & 0 & 0
\end{array}\right)
$$


Therefore, the group ranking of all feasible alternatives $A_{i}(i=1,2,3,4,5)$ is determined as $A_{3} \succ A_{2} \succ A_{4} \succ A_{5} \succ A_{1}$, and the best alternative is $A_{3}$.

\subsection{Comparison with the Aggregation Operator-Based Method}

To show the effectiveness of the proposed method in handling hesitant fuzzy 2dimension linguistic MCGDM problems, the aggregation operator-based hesitant fuzzy 2-dimension linguistic MCGDM method [25] was used to solve the above case related to energy policy selection. The aggregation operator-based method is an easy method. With this method, all individual hesitant fuzzy 2-dimension linguistic decision matrices are first aggregated into a collective hesitant fuzzy 2-dimension linguistic decision matrix. Then, the aggregated decision matrix is used to make the final decision. The steps of the aggregation operator-based method are shown as follows:

Step 1. Utilize the generalized hesitant fuzzy 2-dimension linguistic ordered weighted average (G-HF2DLOWA) operator to aggregate all individual hesitant fuzzy 2-dimension linguistic decision matrices $\hat{H}_{S}^{(p)}=\left(\hat{h}_{S_{i j}}^{(p)}\right)_{5 \times 4}(p=1,2,3)$ shown in Table 1 into a collective hesitant fuzzy 2-dimension linguistic decision matrix $\hat{H}_{S}=\left(\hat{h} S_{i j}\right)_{5 \times 4}$, which is shown in Table 5.

Step 2. Utilize the generalized hesitant fuzzy 2-dimension linguistic weighted average (G-HF2DLWA) operator to aggregate the evaluation values $\hat{h}_{S_{i j}}$ of alternative $A_{i}$ on all attributes (suppose $\lambda=1$ ), and derive the overall evaluation values $\hat{h}_{S_{i}}$ of alternative $A_{i}=(i=1,2,3,4,5)$. In the aggregation operator-based method, the weight vector of the criteria is given by DMs subjectively as $w=(0.3,0.4,0.1,0.2)^{T}$. There are vast amounts of elements in $\hat{h}_{S_{i}}$ represented by an HF2DLE. Taking alternative $A_{1}$ as an example, its overall assessment value is composed of 576 (i.e., $8 \times 6 \times 2 \times 6$ ) elements. Due to space limitations, we will not list them in this paper.

Step 3. Calculate the score values of the overall evaluation values $\hat{h}_{S_{i}}(i=1,2,3,4,5)$ by Equation (9), which is shown as follows:

$$
S\left(\hat{h}_{S_{1}}\right)=0.4997, S\left(\hat{h}_{S_{2}}\right)=0.4062, S\left(\hat{h}_{S_{3}}\right)=0.2815, S\left(\hat{h}_{S_{4}}\right)=0.3649, S\left(\hat{h}_{S_{5}}\right)=0.2369
$$

Step 4. According to the descending order of the score values, $S\left(\hat{h}_{S_{i}}\right)$, the ranking of all feasible alternatives $A_{i}(i=1,2,3,4,5)$ is obtained as $A_{1} \succ A_{2} \succ A_{4} \succ A_{3} \succ A_{5}$, and thus, the best alternative is $A_{1}$.

Comparing the aggregation operator-based method with the proposed method, it is easy to observe that the results yielded by these two methods are obviously different. The reasons may be as follows: (1) The proposed method simultaneously considers the preference relations of pairwise comparisons between alternatives and the evaluation values of alternatives, while the aggregation operator-based method only considers the evaluation values of alternatives. (2) The aggregation-based method may lose information in aggregating all individual decision matrices into a collective decision matrix, while the proposed method first determines the individual ranking of alternatives for each DM and then obtains the group ranking of alternatives by synthesizing the individual rankings. (3) The proposed method determines the criterion weights objectively by a bi-objective hesitant fuzzy 2-dimension linguistic programming model, while in the aggregation operator-based method, the criteria weights are given by DMs subjectively in advance. Since the DMs may have incomplete and indeterminant information, the weights given by DMs might be wrong or unreasonable in some cases. In this sense, the proposed method is better because it considers more information in the analysis process and reduces the influence of subjective factors on final decision results. 
Table 5. Collective hesitant fuzzy 2-dimension linguistic decision matrix.

\begin{tabular}{|c|c|c|c|c|}
\hline Alternatives & $C_{1}$ & $C_{2}$ & $C_{3}$ & $C_{4}$ \\
\hline$A_{1}$ & $\begin{array}{l}\left\{\left(\dot{s}_{4.3182}, \ddot{s}_{3}\right),\left(\dot{s}_{4.5858}, \ddot{s}_{2}\right),\right. \\
\left(\dot{s}_{4.8108}, \ddot{s}_{2}\right),\left(\dot{s}_{5.0000}, \ddot{s}_{2}\right), \\
\left(\dot{s}_{6.0000}, \ddot{s}_{4}\right),\left(\dot{s}_{6.0000}, \ddot{s}_{2}\right), \\
\left.\left(\dot{s}_{6.0000}, \ddot{s}_{2}\right),\left(\dot{s}_{6.0000}, \ddot{s}_{2}\right)\right\}\end{array}$ & $\begin{array}{l}\left\{\left(\dot{s}_{3.5505}, \ddot{s}_{3}\right),\left(\dot{s}_{3.7205}, \ddot{s}_{4}\right),\right. \\
\left(\dot{s}_{3.9402}, \ddot{s}_{1}\right),\left(\dot{s}_{4.0000}, \ddot{s}_{2}\right), \\
\left.\left(\dot{s}_{4.1388}, \ddot{s}_{2}\right),\left(\dot{s}_{4.3182}, \ddot{s}_{1}\right)\right\}\end{array}$ & $\left\{\left(\dot{s}_{3.7205}, \ddot{s}_{4}\right),\left(\dot{s}_{4.1388}, \ddot{s}_{3}\right)\right\}$ & $\begin{array}{l}\left\{\left(\dot{s}_{3.6216}, \ddot{s}_{4}\right),\left(\dot{s}_{3.7866}, \ddot{s}_{5}\right),\right. \\
\left(\dot{s}_{4.0000}, \ddot{s}_{2}\right),\left(\dot{s}_{4.3182}, \ddot{s}_{3}\right), \\
\left.\left(\dot{s}_{4.4349}, \ddot{s}_{3}\right),\left(\dot{s}_{4.5858}, \ddot{s}_{2}\right)\right\}\end{array}$ \\
\hline$A_{2}$ & $\begin{array}{l}\left\{\left(\dot{s}_{2.6563}, \ddot{s}_{5}\right),\left(\dot{s}_{2.8377}, \ddot{s}_{3}\right)\right. \\
\left.\left(\dot{s}_{3.0093}, \ddot{s}_{3}\right),\left(\dot{s}_{3.1716}, \ddot{s}_{3}\right)\right\}\end{array}$ & $\begin{array}{l}\left\{\left(\dot{s}_{4.5858}, \ddot{s}_{4}\right),\left(\dot{s}_{5.0000}, \ddot{s}_{3}\right),\right. \\
\left(\dot{s}_{6.0000}, \ddot{s}_{3}\right),\left(\dot{s}_{6.0000}, \ddot{s}_{3}\right), \\
\left(\dot{s}_{6.0000}, \ddot{s}_{4}\right),\left(\dot{s}_{6.0000}, \ddot{s}_{3}\right) \\
\left(\dot{s}_{6.0000}, \ddot{s}_{4}\right),\left(\dot{s}_{6.0000}, \ddot{s}_{3}\right) \\
\left(\dot{s}_{6.0000}, \ddot{s}_{3}\right),\left(\dot{s}_{6.0000}, \ddot{s}_{3}\right) \\
\left.\left(\dot{s}_{6.0000}, \ddot{s}_{4}\right),\left(\dot{s}_{6.0000}, \ddot{s}_{3}\right)\right\}\end{array}$ & $\begin{array}{l}\left\{\left(\dot{s}_{3.0093}, \ddot{s}_{3}\right),\left(\dot{s}_{3.1716}, \ddot{s}_{4}\right),\right. \\
\left(\dot{s}_{3.4100}, \ddot{s}_{3}\right),\left(\dot{s}_{3.5505}, \ddot{s}_{4}\right), \\
\left(\dot{s}_{6.0000}, \ddot{s}_{3}\right),\left(\dot{s}_{6.0000}, \ddot{s}_{3}\right), \\
\left.\left(\dot{s}_{6.0000}, \ddot{s}_{3}\right),\left(\dot{s}_{6.0000}, \ddot{s}_{3}\right)\right\}\end{array}$ & $\begin{array}{l}\left\{\left(\dot{s}_{4.2679}, \ddot{s}_{3}\right),\left(\dot{s}_{4.4349}, \ddot{s}_{3}\right),\right. \\
\left.\left(\dot{s}_{4.4349}, \ddot{s}_{3}\right),\left(\dot{s}_{4.5858}, \ddot{s}_{3}\right)\right\}\end{array}$ \\
\hline$A_{3}$ & $\begin{array}{l}\left\{\left(\dot{s}_{2.0640}, \ddot{s}_{1}\right),\left(\dot{s}_{2.2776}, \ddot{s}_{1}\right)\right. \\
\left(\dot{s}_{2.4434}, \ddot{s}_{1}\right),\left(\dot{s}_{2.5359}, \ddot{s}_{1}\right) \\
\left(\dot{s}_{2.5913}, \ddot{s}_{2}\right),\left(\dot{s}_{2.6364}, \ddot{s}_{1}\right) \\
\left(\dot{s}_{2.7763}, \ddot{s}_{3}\right),\left(\dot{s}_{2.8698}, \ddot{s}_{1}\right) \\
\left(\dot{s}_{2.9199}, \ddot{s}_{1}\right),\left(\dot{s}_{3.0000}, \ddot{s}_{4}\right) \\
\left.\left(\dot{s}_{3.0870}, \ddot{s}_{1}\right),\left(\dot{s}_{3.2892}, \ddot{s}_{1}\right)\right\}\end{array}$ & $\left\{\left(\dot{s}_{4.6839}, \ddot{s}_{2}\right)\right\}$ & $\begin{array}{l}\left\{\left(\dot{s}_{3.0870}, \ddot{s}_{4}\right),\left(\dot{s}_{3.2892}, \ddot{s}_{1}\right)\right. \\
\left(\dot{s}_{3.5505}, \ddot{s}_{5}\right),\left(\dot{s}_{3.6216}, \ddot{s}_{4}\right) \\
\left(\dot{s}_{3.7205}, \ddot{s}_{1}\right),\left(\dot{s}_{3.7866}, \ddot{s}_{1}\right) \\
\left(\dot{s}_{4.0000}, \ddot{s}_{4}\right),\left(\dot{s}_{4.1388}, \ddot{s}_{1}\right) \\
\left(\dot{s}_{4.3182}, \ddot{s}_{3}\right),\left(\dot{s}_{4.4349}, \ddot{s}_{1}\right) \\
\left.\left(\dot{s}_{4.5858}, \ddot{s}_{3}\right),\left(\dot{s}_{4.6839}, \ddot{s}_{1}\right)\right\}\end{array}$ & $\begin{array}{l}\left\{\left(\dot{s}_{3.4851}, \ddot{s}_{0}\right),\left(\dot{s}_{3.6216}, \ddot{s}_{0}\right),\right. \\
\left.\left(\dot{s}_{4.2217}, \ddot{s}_{3}\right),\left(\dot{s}_{4.3182}, \ddot{s}_{2}\right)\right\}\end{array}$ \\
\hline$A_{4}$ & $\left\{\left(\dot{s}_{4.3182}, \ddot{s}_{4}\right),\left(\dot{s}_{4.4349}, \ddot{s}_{1}\right)\right\}$ & $\begin{array}{l}\left\{\left(\dot{s}_{2.4324}, \ddot{s}_{4}\right),\left(\dot{s}_{2.5913}, \ddot{s}_{2}\right),\right. \\
\left(\dot{s}_{2.7763}, \ddot{s}_{2}\right),\left(\dot{s}_{2.9199}, \ddot{s}_{2}\right), \\
\left(\dot{s}_{3.0870}, \ddot{s}_{2}\right),\left(\dot{s}_{3.2168}, \ddot{s}_{2}\right) \\
\left.\left(\dot{s}_{3.3679}, \ddot{s}_{2}\right),\left(\dot{s}_{3.4851}, \ddot{s}_{2}\right)\right\}\end{array}$ & $\begin{array}{l}\left\{\left(\dot{s}_{4.2217}, \ddot{s}_{3}\right),\left(\dot{s}_{4.3182}, \ddot{s}_{3}\right)\right. \\
\left(\dot{s}_{4.4349}, \ddot{s}_{2}\right),\left(\dot{s}_{4.5047}, \ddot{s}_{3}\right) \\
\left.\left(\dot{s}_{4.5858}, \ddot{s}_{3}\right),\left(\dot{s}_{4.6839}, \ddot{s}_{2}\right)\right\}\end{array}$ & $\begin{array}{l}\left\{\left(\dot{s}_{2.9199}, \ddot{s}_{4}\right),\left(\dot{s}_{3.0870}, \ddot{s}_{4}\right),\right. \\
\left(\dot{s}_{3.2892}, \ddot{s}_{2}\right),\left(\dot{s}_{3.4851}, \ddot{s}_{3}\right), \\
\left.\left(\dot{s}_{3.6216}, \ddot{s}_{3}\right),\left(\dot{s}_{3.7866}, \ddot{s}_{2}\right)\right\}\end{array}$ \\
\hline$A_{5}$ & $\begin{array}{l}\left\{\left(\dot{s}_{4.5858}, \ddot{s}_{3}\right),\left(\dot{s}_{4.8108}, \ddot{s}_{3}\right)\right. \\
\left.\left(\dot{s}_{6.0000}, \ddot{s}_{1}\right),\left(\dot{s}_{6.0000}, \ddot{s}_{1}\right)\right\}\end{array}$ & $\begin{array}{l}\left\{\left(\dot{s}_{4.1388}, \ddot{s}_{4}\right),\left(\dot{s}_{4.2217}, \ddot{s}_{3}\right),\right. \\
\left(\dot{s}_{4.4349}, \ddot{s}_{3}\right),\left(\dot{s}_{4.5047}, \ddot{s}_{3}\right), \\
\left(\dot{s}_{6.0000}, \ddot{s}_{3}\right),\left(\dot{s}_{6.0000}, \ddot{s}_{3}\right), \\
\left.\left(\dot{s}_{6.0000}, \ddot{s}_{3}\right),\left(\dot{s}_{6.0000}, \ddot{s}_{3}\right)\right\}\end{array}$ & $\begin{array}{l}\left\{\left(\dot{s}_{3.2892}, \ddot{s}_{0}\right),\left(\dot{s}_{3.7205}, \ddot{s}_{2}\right)\right. \\
\left(\dot{s}_{3.7866}, \ddot{s}_{0}\right),\left(\dot{s}_{4.1388}, \ddot{s}_{3}\right) \\
\left.\left(\dot{s}_{6.0000}, \ddot{s}_{2}\right),\left(\dot{s}_{6.0000}, \ddot{s}_{2}\right)\right\}\end{array}$ & $\left\{\left(\dot{s}_{2.0640}, \ddot{s}_{4}\right),\left(\dot{s}_{2.2776}, \ddot{s}_{3}\right)\right\}$ \\
\hline
\end{tabular}

\subsection{Comparison with the Hesitant Fuzzy Linguistic LINMAP Method}

To show the effectiveness of the proposed method in adopting HF2DLEs to preserve the DMs' hesitant qualitative preference information, we compared the results obtained by the hesitant fuzzy linguistic LINMAP method [33] and the proposed method. In the hesitant fuzzy linguistic LINMAP method [33], the evaluation values of alternatives are denoted by HFLEs, and the truth degrees of alternative comparisons are denoted by crisp numbers in $[0,1]$. Thus, the individual hesitant fuzzy 2-dimension linguistic decision matrices $\hat{H}_{S}^{(p)}=\left(\hat{h}_{S_{i j}}^{(p)}\right)_{5 \times 4}$ shown in Table 1 should be transformed into individual hesitant fuzzy linguistic decision matrices $H_{S}^{(p)}=\left(h_{S_{i j}}^{(p)}\right)_{5 \times 4}(p=1,2,3)$ by only extracting the first dimension linguistic information in HF2DLEs, as shown in Table 6, and the hesitant fuzzy 2-dimension linguistic truth degrees of alternative comparisons are transformed into crisp numbers with score function of HF2DLE, which are shown as follows:

$$
\begin{aligned}
\Omega^{1}= & \{<(2,1), 0.1944>,<(1,3), 0.2777>,<(5,4), 0.3519>,<(2,5), 0.1389>, \\
& <(3,2), 0.4450>,<(3,4), 0.2501>\}, \\
\Omega^{2}= & \{<(1,2), 0.3889>,<(3,1), 0.4444>,<(4,5), 0.2361>,<(5,2), 0>, \\
& <(2,3), 0.2361>,<(4,3), 0.3194>\}, \\
\Omega^{3}=\{< & (2,4), 0.4167>,<(1,4), 0.2083>,<(5,1), 0.0417>,<(5,3), 0.0833>\} .
\end{aligned}
$$


Table 6. Individual hesitant fuzzy linguistic decision matrices.

\begin{tabular}{cccccc}
\hline DMs & Alternatives & $C_{1}$ & $C_{2}$ & $C_{3}$ & $C_{4}$ \\
\hline$A_{1}$ & $\left\{\dot{s}_{4}, \dot{s}_{5}\right\}$ & $\left\{\dot{s}_{3}, \dot{s}_{4}\right\}$ & $\left\{\dot{s}_{5}\right\}$ & $\left\{\dot{s}_{2}, \dot{s}_{3}, \dot{s}_{4}\right\}$ \\
$D_{1}$ & $A_{2}$ & $\left\{\dot{s}_{1}, \dot{s}_{2}\right\}$ & $\left\{\dot{s}_{5}, \dot{s}_{6}\right\}$ & $\left\{\dot{s}_{2}, \dot{s}_{3}\right\}$ & $\left\{\dot{s}_{3}, \dot{s}_{4}\right\}$ \\
& $A_{3}$ & $\left\{\dot{s}_{2}, \dot{s}_{3}\right\}$ & $\left\{\dot{s}_{3}\right\}$ & $\left\{\dot{s}_{3}, \dot{s}_{4}, \dot{s}_{5}\right\}$ & $\left\{\dot{s}_{4}\right\}$ \\
& $A_{4}$ & $\left\{\dot{s}_{4}\right\}$ & $\left\{\dot{s}_{3}, \dot{s}_{4}\right\}$ & $\left\{\dot{s}_{4}, \dot{s}_{5}\right\}$ & $\left\{\dot{s}_{1}, \dot{s}_{2}, \dot{s}_{3}\right\}$ \\
& $A_{5}$ & $\left\{\dot{s}_{5}, \dot{s}_{6}\right\}$ & $\left\{\dot{s}_{0}, \dot{s}_{1}\right\}$ & $\left\{\dot{s}_{3}\right\}$ & $\left\{\dot{s}_{1}, \dot{s}_{2}\right\}$ \\
\hline$A_{1}$ & $\left\{\dot{s}_{5}, \dot{s}_{6}\right\}$ & $\left\{\dot{s}_{5}\right\}$ & $\left\{\dot{s}_{3}, \dot{s}_{4}\right\}$ & $\left\{\dot{s}_{4}, \dot{s}_{5}\right\}$ \\
& $A_{2}$ & $\left\{\dot{s}_{1}, \dot{s}_{2}\right\}$ & $\left\{\dot{s}_{4}, \dot{s}_{5}, \dot{s}_{6}\right\}$ & $\left\{\dot{s}_{5}, \dot{s}_{6}\right\}$ & $\left\{\dot{s}_{5}\right\}$ \\
$D_{2}$ & $A_{3}$ & $\left\{\dot{s}_{1}, \dot{s}_{2}, \dot{s}_{3}\right\}$ & $\left\{\dot{s}_{5}\right\}$ & $\left\{\dot{s}_{4}, \dot{s}_{5}\right\}$ & $\left\{\dot{s}_{1}, \dot{s}_{2}\right\}$ \\
& $A_{4}$ & $\left\{\dot{s}_{5}\right\}$ & $\left\{\dot{s}_{3}, \dot{s}_{4}\right\}$ & $\left\{\dot{s}_{5}\right\}$ & $\left\{\dot{s}_{3}, \dot{s}_{4}\right\}$ \\
& $A_{5}$ & $\left\{\dot{s}_{4}, \dot{s}_{5}\right\}$ & $\left\{\dot{s}_{5}, \dot{s}_{6}\right\}$ & $\left\{\dot{s}_{3}, \dot{s}_{4}\right\}$ & $\left\{\dot{s}_{3}\right\}$ \\
\hline$D_{3}$ & $\left\{\dot{s}_{4}, \dot{s}_{5}\right\}$ & $\left\{\dot{s}_{2}, \dot{s}_{3}, \dot{s}_{4}\right\}$ & $\left\{\dot{s}_{3}\right\}$ & $\left\{\dot{s}_{4}\right\}$ \\
& $A_{1}$ & $\left\{\dot{s}_{5}\right\}$ & $\left\{\dot{s}_{5}, \dot{s}_{6}\right\}$ & $\left\{\dot{s}_{1}, \dot{s}_{2}\right\}$ & $\left\{\dot{s}_{3}, \dot{s}_{4}\right\}$ \\
& $A_{2}$ & $\left\{\dot{s}_{3}, \dot{s}_{4}\right\}$ & $\left\{\dot{s}_{5}\right\}$ & $\left\{\dot{s}_{2}, \dot{s}_{3}\right\}$ & $\left\{\dot{s}_{4}, \dot{s}_{5}\right\}$ \\
& $A_{3}$ & $\left\{\dot{s}_{2}, \dot{s}_{3}\right\}$ & $\left\{\dot{s}_{0}, \dot{s}_{1}\right\}$ & $\left\{\dot{s}_{1}, \dot{s}_{2}, \dot{s}_{3}\right\}$ & $\left\{\dot{s}_{4}\right\}$ \\
$A_{4}$ & $\left\{\dot{s}_{4}\right\}$ & $\left\{\dot{s}_{4}, \dot{s}_{5}\right\}$ & $\left\{\dot{s}_{4}, \dot{s}_{5}, \dot{s}_{6}\right\}$ & $\left\{\dot{s}_{2}\right\}$ \\
\hline & $A_{5}$ & & &
\end{tabular}

First, we use the hesitant fuzzy linguistic weighted average (HFLWA) operator to aggregate all individual hesitant fuzzy linguistic decision matrices $H_{S}^{(p)}=\left(h_{S_{i j}}^{(p)}\right)_{5 \times 4}(p=1,2,3)$ into a collective hesitant fuzzy linguistic decision matrix $H_{S}=\left(h_{S_{i j}}\right)_{5 \times 4}$, which is shown in Table 7.

Table 7. Collective hesitant fuzzy linguistic decision matrix.

\begin{tabular}{|c|c|c|c|c|}
\hline Alternatives & $C_{1}$ & $C_{2}$ & $C_{3}$ & $C_{4}$ \\
\hline$A_{1}$ & $\left\{\dot{S}_{4.3333}, \dot{S}_{4.8333}, \dot{S}_{5.3333}\right\}$ & $\left\{\dot{s}_{3.3333}, \dot{s}_{3.8333}, \dot{s}_{4.3333}\right\}$ & $\left\{\dot{s}_{3.6667}, \dot{s}_{3.8333}, \dot{S}_{4}\right\}$ & $\left\{\dot{s}_{3.3333}, \dot{s}_{3.8333}, \dot{s}_{4.3333}\right\}$ \\
\hline$A_{2}$ & $\left\{\dot{s}_{2.3333}, \dot{s}_{2.6667}, \dot{s}_{3}\right\}$ & $\left\{\dot{s}_{4.6667}, \dot{s}_{5.3333}, \dot{s}_{6}\right\}$ & $\left\{\dot{s}_{2.6667}, \dot{s}_{3.1667}, \dot{s}_{3.6667}\right\}$ & $\left\{\dot{s}_{3.6667}, \dot{s}_{4}, \dot{s}_{4.3333}\right\}$ \\
\hline$A_{3}$ & $\left\{\dot{s}_{2}, \dot{s}_{2.6667}, \dot{s}_{3.3333}\right\}$ & $\left\{\dot{s}_{4.3333}, \dot{s}_{4.3333}, \dot{s}_{4.3333}\right\}$ & $\left\{\dot{s}_{3}, \dot{s}_{3.6667}, \dot{s}_{4.3333}\right\}$ & $\left\{\dot{s}_{3}, \dot{s}_{3.3333}, \dot{s}_{3.6667}\right\}$ \\
\hline$A_{4}$ & $\left\{\dot{s}_{3.6667}, \dot{s}_{3.8333}, \dot{s}_{4}\right\}$ & $\left\{\dot{s}_{2}, \dot{s}_{2.5}, \dot{s}_{3}\right\}$ & $\left\{\dot{s}_{3.3333}, \dot{s}_{3.8333}, \dot{s}_{4.3333}\right\}$ & $\left\{\dot{s}_{2.6667}, \dot{s}_{3.1667}, \dot{s}_{3.6667}\right\}$ \\
\hline$A_{5}$ & $\left\{\dot{s}_{4.3333}, \dot{s}_{4.6667}, \dot{s}_{5}\right\}$ & $\left\{\dot{s}_{3}, \dot{s}_{3.5}, \dot{s}_{4}\right\}$ & $\left\{\dot{s}_{3.3333}, \dot{s}_{3.8333}, \dot{s}_{4.3333}\right\}$ & $\left\{\dot{s}_{2}, \dot{s}_{2.1667}, \dot{s}_{2.3333}\right\}$ \\
\hline
\end{tabular}

Taking $\widetilde{q}=0.001$, by constructing the group inconsistency and consistency indices based on the distance measure between each alternative and PIS, a hesitant fuzzy linguistic programming model is constructed by minimizing the group inconsistency index. Using the developed solution method for the hesitant fuzzy linguistic programming model, the main components of the optimal solution can be obtained as follows:

$$
\begin{gathered}
w_{1}=0.5336, w_{2}=0.4141, w_{3}=0.0208, w_{4}=0.0315 \\
h_{S_{1}}^{+}=\left\{\dot{s}_{2.1434}, \dot{s}_{3.7434}, \dot{s}_{4.9451}\right\}, h_{S_{2}}^{+}=\left\{\dot{s}_{1.5196}, \dot{s}_{3.2577}, \dot{s}_{5.0687}\right\} \\
h_{S_{3}}^{+}=\left\{\left(\dot{s}_{1.5132}, \dot{s}_{3.3163}, \dot{s}_{4.8806}\right\}, h_{S_{4}}^{+}=\left\{\dot{s}_{1.1709}, \dot{s}_{2.7938}, \dot{s}_{4.5785}\right\} .\right.
\end{gathered}
$$

Then, the distances between alternatives $A_{i}(i=1,2,3,4,5)$ and PIS can be obtained as $d_{1}=0.1980, d_{2}=0.3369, d_{3}=0.2146, d_{4}=0.1377$, and $d_{5}=0.1729$. Therefore, according to the ascending order of distances, the ranking of all feasible alternatives is obtained as $A_{4} \succ A_{5} \succ A_{1} \succ A_{3} \succ A_{2}$, and the best alternative is $A_{4}$.

It can be inferred that the results obtained by the hesitant fuzzy linguistic LINMAP method and the proposed method are obviously different, i.e., the best alternative obtained by the proposed method is $A_{3}$, while the best alternative obtained by the hesitant fuzzy linguistic LINMAP method is $A_{4}$. In the hesitant fuzzy linguistic LINMAP method, the crisp numbers and HFLEs are, respectively, used to express the truth degrees of alternative comparisons and the evaluation values of alternatives. Due to the fuzzy or uncertain information in decision making and the hesitation of human thinking, it may be impossible 
for DMs to use crisp numbers to provide the truth degrees of alternative comparisons, and the importance of linguistic terms in an HFLE are different. Considering the flexibility of HF2DLTS in reflecting DMs' hesitant qualitative preference information, this paper adopted HF2DLEs to provide the evaluation values of alternatives and the truth degrees of alternative comparisons. Since the HF2DLEs can be converted into HFLEs or crisp numbers, the latter can be regarded as special cases of the former.

\subsection{Comparison with Other Existing MCGDM Methods}

To further demonstrate the effectiveness of the proposed method, we compare other methods $[58,59]$ with our proposed method in this subsection.

(1) Riaz et al. [58] proposed novel methods for solving MCGDM problems in which the evaluation values of alternatives are denoted as hesitant fuzzy soft set (HFSset). Xu et al. [59] investigated a missing value estimation and consensus analysis method for incomplete preference relations expressed by an unordered hesitant fuzzy element (HFE). No matter whether it is an HFS set or unordered HFE, it uses a set of numerical values to express the possible degrees of an alternative satisfying the parameter specified or the preference degrees of pairwise comparisons between alternatives. Due to the increasing vagueness and uncertainty in decision making, numerical values may be inadequate to describe these degrees. Compared with other methods [58,59], our proposed method of adopting HF2DLEs to depict both the evaluation values of alternatives and the truth degrees of alternative comparisons is capable of better modeling the real-life MCGDM problems, especially under the hesitant linguistic environment.

(2) The methods proposed in [58,59] either only consider the evaluation values of alternatives or only consider the preference relations of alternative comparisons, while our proposed method takes both evaluation values of alternatives and preference relations of alternative comparisons.

(3) In the method proposed in [58], criteria weights should be given in advance. If criteria weights are completely unknown or partially known, the method cannot be used. However, in our proposed method, there is no need to give criteria weights in advance, and criteria weights can be determined objectively by constructing a programming model.

(4) If the preference relations are incomplete in the method proposed in [59], we should estimate the missing elements based on multiplicative consistency to complement preference relations before consensus analysis, while our proposed method can be directly used to handle the incomplete or even non-transitive preference relations.

\section{Conclusions}

HF2DLTS is a powerful technique to represent the DM's hesitant qualitative preference information, especially in some complicated MCGDM problems. In this paper, a novel fuzzy programming method was proposed to solve MCGDM problems in which the evaluation values of alternatives and the truth degrees of pairwise comparisons between alternatives were denoted by HF2DLEs, and the preferences over alternatives and criteria were incomplete. To obtain the RCDs of all alternatives to HF2DLPIS for each DM, the Euclidean distance measure between HF2DLEs was defined. Then, according to RCDs and preference relations on alternatives, the hesitant fuzzy 2-dimension linguistic group consistency and inconsistency indices were constructed, respectively, based on which a bi-objective hesitant fuzzy 2-dimension linguistic programming model was constructed by minimizing the group inconsistency index, as well as maximizing the group consistency index, to determine criteria weights, HF2DLPIS and HF2DLNIS, objectively. Furthermore, the individual ranking of alternatives furnished by each DM was subsequently obtained according to the descending order of RCDs, and to generate the group ranking of alternatives, a single-objective assignment model was established by minimizing the deviations between individual rankings and the group ranking, and thus, the most desirable alter- 
native was determined. Finally, a case study about energy policy selection was provided to illustrate the applicability of the proposed method. Some comparison analyses with the aggregation operator-based hesitant fuzzy 2-dimension linguistic MCGDM method, the hesitant fuzzy linguistic LINMAP method, and other existing MCGDM methods were conducted to demonstrate the effectiveness of the proposed method.

The main features of the proposed method are that both the truth degrees of pairwise comparisons between alternatives and the evaluation values of alternatives given by DMs are formulated as HF2DLEs, and criteria weights, HF2DLPIS and HF2DLNIS, are obtained objectively instead of being given subjectively in advance. However, the proposed method also has some limitations: If there are too many criteria or alternatives, the size of the hesitant fuzzy 2-dimension linguistic programming model might be quite large, but some software packages can be used to solve it. In future research, it is interesting to consider how to construct the programming model for hesitant fuzzy 2-dimension linguistic MCGDM problems with low consensus degree or criteria interactions. Additionally, we will apply the proposed method to solve other complicated decision-making problems, such as supplier selection, risk investment, healthcare management, etc.

Author Contributions: Conceptualization, X.L. and D.J.; methodology, X.L.; software, D.J.; writingoriginal draft preparation, X.L.; writing - review and editing, D.J. All authors have read and agreed to the published version of the manuscript.

Funding: This research was funded by "the Fundamental Research Funds for the Central Universities" in UIBE(17QN01, 20YQ04), MOE (Ministry of Education of China) Youth Fund Project of Humanities and Social Sciences (19YJC630107), and "Program for Young Excellent Talents" in UIBE(20YQ12).

Institutional Review Board Statement: Not applicable.

Informed Consent Statement: Not applicable.

Conflicts of Interest: The authors declare no conflict of interest.

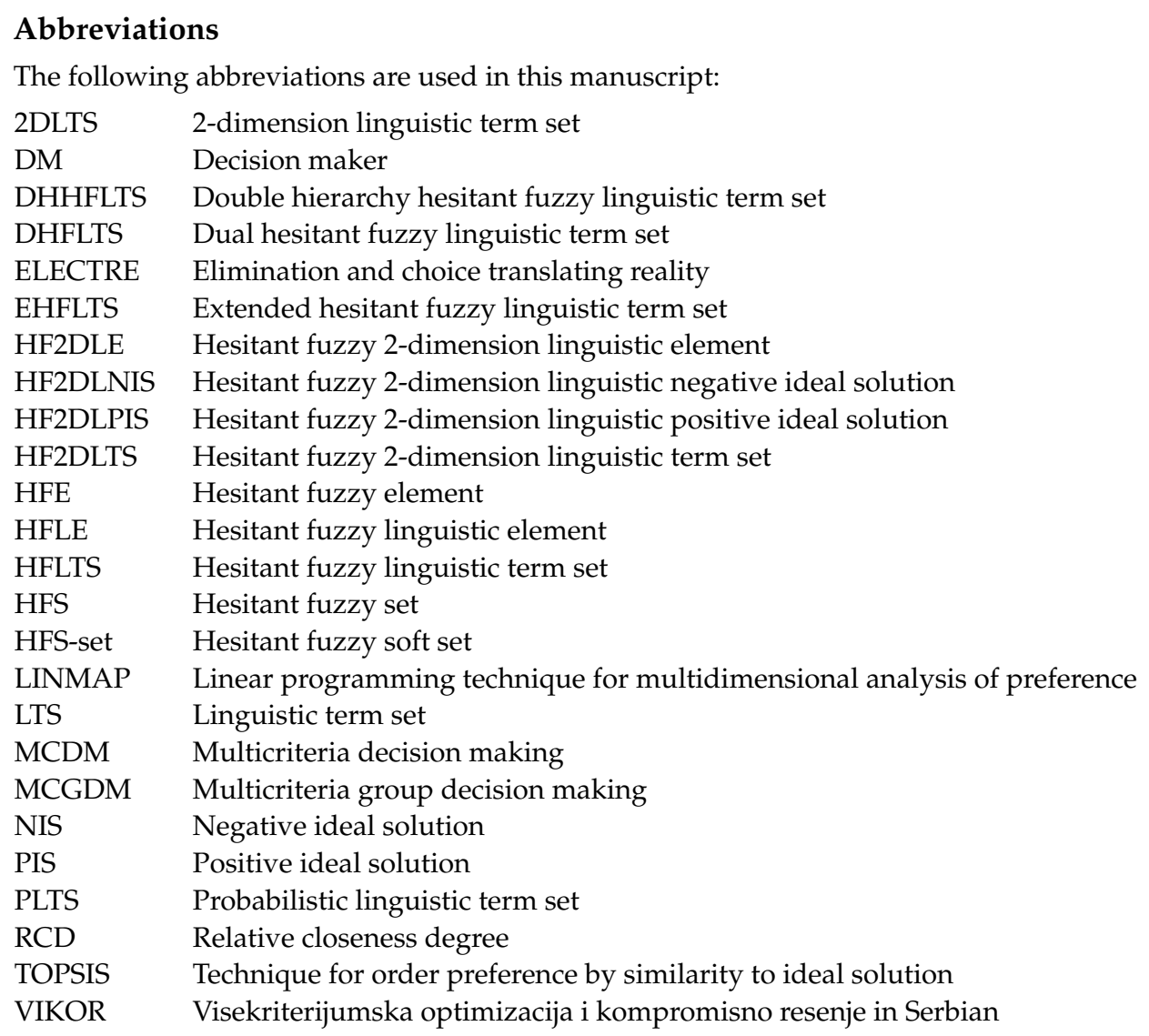




\section{References}

1. Zadeh, L.A. The concept of a linguistic variable and its applications to approximate reasoning-Part I. Inf. Sci. 1975, 8, 199-249. [CrossRef]

2. Herrera, F.; Herrera-Viedma, E.; Martínez, L. A fuzzy linguistic methodology to deal with unbalanced linguistic term sets. IEEE Trans. Fuzzy Syst. 2008, 16, 354-370.

3. Rodriguez, R.; Martínez, L. An analysis of symbolic linguistic computing models in decision making. Int. J. Gen. Syst. 2013, 42, 121-136. [CrossRef]

4. Torra, V. Hesitant fuzzy sets. Int. J. Intell. Syst. 2010, 25, 529-539. [CrossRef]

5. Rodríguez, R.M.; Martínez, L.; Herrera, F. Hesitant fuzzy linguistic term sets for decision making. IEEE Trans. Fuzzy Syst. 2012, 20, 109-119. [CrossRef]

6. Liao, H.C.; Xu, Z.S.; Herrera-Viedma, E.; Herrera, F. Hesitant fuzzy linguistic term set and its application in decision making: A state-of-the-art survey. Int. J. Fuzzy Syst. 2018, 20, 2084-2110. [CrossRef]

7. Gou, X.J.; Xu, Z.S. Novel basic operational laws for linguistic terms, hesitant fuzzy linguistic term sets and probabilistic linguistic term sets. Inf. Sci. 2016, 372, 407-427. [CrossRef]

8. Lee, L.W.; Chen, S.M. Fuzzy decision making based on likelihood-based comparison relations of hesitant fuzzy linguistic term sets and hesitant fuzzy linguistic operators. Inf. Sci. 2015, 294, 513-529. [CrossRef]

9. Wei, C.P.; Zhao, N.; Tang, X.J. Operators and comparisons of hesitant fuzzy linguistic term sets. IEEE Trans. Fuzzy Syst. 2014, 22, 575-586. [CrossRef]

10. Liao, H.C.; Xu, Z.S. Approaches to manage hesitant fuzzy linguistic information based on the cosine distance and similarity measures for HFLTSs and their application in qualitative decision making. Expert Syst. Appl. 2015, 42, 5328-5336.

11. Liao, H.C.; Xu, Z.S.; Zeng, X.J. Distance and similarity measures for hesitant fuzzy linguistic term sets and their application in multi-criteria decision making. Inf. Sci. 2014, 271, 125-142. [CrossRef]

12. Liao, H.C.; Xu, Z.S.; Zeng, X.J.; Merigó, J.M. Qualitative decision making with correlation coefficients of hesitant fuzzy linguistic term sets. Knowl.-Based Syst. 2015, 76, 127-138. [CrossRef]

13. Gou, X.J.; Xu, Z.S.; Liao, H.C. Multi-criteria decision making based on bonferroni means with hesitant fuzzy linguistic information. Soft Comput. 2017, 21, 6515-6529. [CrossRef]

14. Xu, Y.J.; Xu, A.W.; Merigó, J.M.; Wang, H.M. Hesitant fuzzy linguistic ordered weighted distance operators for group decision making. J. Appl. Math. Comput. 2015, 49, 285-308. [CrossRef]

15. Farhadinia, B. Multiple criteria decision-making methods with completely unknown weights in hesitant fuzzy linguistic term setting. Knowl.-Based Syst. 2016, 93, 135-141. [CrossRef]

16. Liao, H.C.; Xu, Z.S.; Zeng, X.J. Hesitant fuzzy linguistic VIKOR method and its application in qualitative multiple criteria decision making. IEEE Trans. Fuzzy Syst. 2015, 23, 1343-1355. [CrossRef]

17. Wei, C.P.; Ren, Z.; Rodríguez, R.M. A hesitant fuzzy linguistic TODIM method based on score function. Int. J. Comput. Intell. Syst. 2015, 8, 701-712. [CrossRef]

18. Liu, H.B.; Cai, J.F.; Jiang, L. On improving the additive consistency of the fuzzy preference relations based on comparative linguistic expressions. Int. J. Intell. Syst. 2014, 29, 544-559. [CrossRef]

19. Zhang, Z.M.; Wu, C. On the use of multiplicative consistency in hesitant fuzzy linguistic preference relations. Knowl.-Based Syst. 2014, 72, 13-27. [CrossRef]

20. Zhu, B.; Xu, Z.S. Consistency measures for hesitant fuzzy linguistic preference relations. IEEE Trans. Fuzzy Syst. 2014, 22, 35-45. [CrossRef]

21. Wang, H. Extended hesitant fuzzy linguistic term sets and their aggregation in group decision making. Int. J. Comput. Intell. Syst. 2015, 8, 14-33.

22. Gou, X.J.; Liao, H.C.; Xu, Z.S.; Herrera, F. Double hierarchy hesitant fuzzy linguistic term set and multimoora method: A case of study to evaluate the implementation status of haze controlling measures. Inf. Fusion 2017, 38, 22-34. [CrossRef]

23. Pang, Q.; Wang, H.; Xu, Z.S. Probabilistic linguistic term sets in multi-attribute group decision making. Inf. Sci. 2016, 369, 128-143. [CrossRef]

24. Zhu, W.; Zhou, G.; Yang, S. An approach to group decision making based on 2-dimension linguistic assessment information. Syst. Eng. 2009, 27, 113-118.

25. Liu, X.Y.; Ju, Y.B.; Qu, Q.Q. Hesitant Fuzzy 2-Dimension Linguistic Term Set and its Application to Multiple Attribute Group Decision Making. Int. J. Fuzzy Syst. 2018, 20, 2301-2321. [CrossRef]

26. Srinivasan, V.; Shocker, A.D. Linear programming techniques for multidimensional analysis of preference. Psychometrica 1973, 38 , 337-342. [CrossRef]

27. Zhang, X.L.; Xu, Z.S. Interval programming method for hesitant fuzzy multi-attribute group decision making with incomplete preference over alternatives. Comput. Ind. Eng. 2014, 75, 217-229. [CrossRef]

28. Chen, T.Y. An interval-valued intuitionistic fuzzy LINMAP method with inclusion comparison possibilities and hybrid averaging operations for multiple criteria group decision making. Knowl.-Based Syst. 2013, 45, 134-146. [CrossRef]

29. Li, D.F.; Chen, G.H.; Huang, Z.G. Linear programming method for multiattribute group decision making using IF sets. Inf. Sci. 2010, 180, 1591-1609. [CrossRef] 
30. Li, D.F.; Yang, J.B. Fuzzy linear programming technique for multiattribute group decision making in fuzzy environments. Inf. Sci. 2004, 158, 263-275. [CrossRef]

31. Zhang, W.K.; Ju, Y.B.; Liu, X.Y. Interval-valued intuitionistic fuzzy programming technique for multicriteria group decision making based on Shapley values and incomplete preference information. Soft Comput. 2016, 21, 1-18. [CrossRef]

32. Zhang, X.L.; Xu, Z.S.; Xing, X.M. Hesitant fuzzy programming technique for multidimensional analysis of hesitant fuzzy preferences. OR Spectrum 2016, 38, 789-817. [CrossRef]

33. Xu, Y.J.; Xu, A.W.; Wang, H.M. Hesitant fuzzy linguistic linear programming technique for multidimensional analysis of preference for multi-attribute group decision making. Int. J. Mach. Learn. Cyber. 2016, 7, 1-11. [CrossRef]

34. Liao, H.C.; Jiang, L.S.; Xu, Z.S.; Xu, J.P.; Herrera, F. A linear programming method for multiple criteria decision making with probabilistic linguistic information. Inf. Sci. 2017, 415-416, 341-355. [CrossRef]

35. Li, D.F.; Sun, T. Fuzzy LINMAP method for multiattribute group decision making with linguistic variables and incomplete information. Int. J. Uncertain. Fuzziness Knowl.-Based Syst. 2007, 15, 153-173. [CrossRef]

36. Qin, J.D.; Liu, X.W.; Pedrycz, W. A multiple attribute interval type-2 fuzzy group decision making and its application to supplier selection with extended LINMAP method. Soft Comput. 2017, 21, 1-20. [CrossRef]

37. Song, W.; Zhu, J.; Zhang, S.; Liu, X. A multi-stage uncertain risk decision making method with reference point based on extended LINMAP method. J. Intell. Fuzzy Syst. 2018, 35, 1133-1146. [CrossRef]

38. Wan, S.P.; Jin, Z.; Dong, J.Y. Pythagorean fuzzy mathematical programming method for multi-attribute group decision making with Pythagorean fuzzy truth degrees. Knowl. Inf. Syst. 2018, 55, 437-466. [CrossRef]

39. Xue, W.; Xu, Z.; Zhang, X.; Tian, X. Pythagorean fuzzy LINMAP method based on the entropy theory for railway project investment decision making. Int. J. Intell. Syst. 2018, 33, 93-125. [CrossRef]

40. Chen, T.Y. Pythagorean fuzzy linear programming technique for multidimensional analysis of preference using a squareddistance-based approach for multiple criteria decision analysis. Expert Syst. Appl. 2021, 164, 113908. [CrossRef]

41. Wan, S.P.; Li, D.F. Fuzzy mathematical programming approach to heterogeneous multi-attribute decision making with intervalvalued intuitionistic fuzzy truth degrees. Inf. Sci. 2015, 325, 484-503. [CrossRef]

42. Wan, S.P.; Qin, Y.L.; Dong, J.Y. A hesitant fuzzy mathematical programming method for hybrid multi-criteria group decision making with hesitant fuzzy truth degrees. Knowl.-Based Syst. 2017, 138, 232-248. [CrossRef]

43. Zhang, W.K.; Yan, Y.B.; Liu, X.Y.; Giannakis, M. A mathematical programming based method for heterogeneous multicriteria group decision analysis with aspirations and incomplete preference information. Comput. Ind. Eng. 2017, 113, 541-557. [CrossRef]

44. Hwang, C.L.; Yoon, K. Multiple Attribute Decision Making: Methods and Applications; Springer: Berlin/Heidelberg, Germany, 1981

45. Zhang, X.F.; Su, J.F. A combined fuzzy DEMATEL and TOPSIS approach for estimating participants in knowledge-intensive crowdsourcing. Comput. Ind. Eng. 2019, 137, 106085. [CrossRef]

46. Wu, Z.B.; Xu, J.P.; Jiang, X.L.; Zhong, L. Two MAGDM models based on hesitant fuzzy linguistic term sets with possibility distributions: VIKOR and TOPSIS. Inf. Sci. 2019, 473, 101-120. [CrossRef]

47. Sun, G.D.; Guan, X.; Yi, X.; Zhou, Z. An innovative TOPSIS approach based on hesitant fuzzy correlationcoefficient and its applications. Appl. Soft Comput. 2018, 68, 249-267. [CrossRef]

48. Herrera, F.; Herrera-Viedma, E. Linguistic decision analysis: Steps for solving decision problems under linguistic information. Fuzzy Sets Syst. 2000, 115, 67-82. [CrossRef]

49. Herrera, F.; Herrera-Viedma, E.; Verdegay, J.L. A model of consensus in group decision making under linguistic assessment. Fuzzy Sets Syst. 1996, 78, 73-87. [CrossRef]

50. Bonissone, P.P.; Decker, K.S. Selecting Uncertainty Calculi and Granularity: An Experiment in Trading-off Precision and Complexity. In Proceedings of the First Annual Conference on Uncertainty in Artificial Intelligence, Los Angeles, CA, USA, 10-12 July 1986.

51. Miller, G.A. The magical number seven, plus or minus two: Some limits on our capacity of processing information. Psychol. Rev. 1994, 101, 343-352. [CrossRef] [PubMed]

52. Xu, Z.S. Deviation measures of linguistic preference relations in group decision making. Omega 2005, 33, 249-254. [CrossRef]

53. Zhang, J.L.; Qi, X.W. Research on multiple attribute decision making under hesitant fuzzy linguistic environment with application to production strategy decision making. Adv. Mat. Res. 2013, 753-755, 2829-2836. [CrossRef]

54. Yang, S.H.; Ju, Y.B. Dual hesitant fuzzy linguistic aggregation operators and their applications to multi-attribute decision making. J. Intell. Syst. 2014, 27, 1935-1947. [CrossRef]

55. Kim, S.H.; Ahn, B.S. Interactive group decision making procedure under incomplete information. Eur. J. Oper. Res. 1999, 116, 498-507. [CrossRef]

56. Park, K.S.; Kim, S.H. Tools for interactive multi-attribute decision making with incompletely identified information. Eur. J. Oper. Res. 1997, 98, 111-123. [CrossRef]

57. Podinovski, V.V. Set choice problems with incomplete information about the preference of the decision makers. Eur. J. Oper. Res. 2010, 207, 371-379. [CrossRef]

58. Riaz, M.; Davvaz, B.; Fakhar, A.; Firdous, A. Hesitant fuzzy soft topology and its application to multi-attribute group decision making. Soft Comput. 2020, 24, 16269-16289. [CrossRef]

59. Xu, Y.J.; Li, C.Y.; Wen, X.W. Missing values estimation and consensus building for incomplete hesitant fuzzy preference relations with multiplicative consistency. Int. J. Comput. Int. Syst. 2018, 11, 101-119. [CrossRef] 